\title{
A chronological and geochemical study of the Tadamigawa older-stage granites: Igneous activity in the west of the Tanakura Tectonic Line (TTL) of northeastern Japan
}

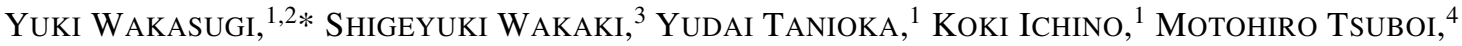 \\ YOSHIHIRO ASAHARA ${ }^{5}$ and ATSUSHI NODA ${ }^{6}$ \\ ${ }^{1}$ Department of Chemistry, School of Science and Technology, Kwansei Gakuin University, Sanda, Hyogo 669-1337, Japan \\ ${ }^{2}$ Institute for Space-Earth Environmental Research, Nagoya University, Nagoya 464-8601, Japan \\ ${ }^{3}$ Kochi Institute for Core Sample Research, Japan Agency for Marine-Earth Science and Technology, Kochi 783-8502, Japan \\ ${ }^{4}$ Department of Applied Chemistry for Environment, School of Science and Technology, Kwansei Gakuin University, \\ Sanda, Hyogo 669-1337, Japan \\ ${ }^{5}$ Department of Earth and Environmental Sciences, Graduate School of Environmental Studies, Nagoya University, \\ Nagoya 464-8601, Japan \\ ${ }^{6}$ Geological Survey of Japan, National Institute of Advanced Industrial Science Technology, Tsukuba, Ibaraki 305-8567, Japan
}

(Received November 2, 2019; Accepted June 4, 2020)

\begin{abstract}
The Cretaceous to early Paleogene magmatism occurred in the Honshu Arc actively and the formation age for this period shifted regionally in the Southwestern and Northeastern Japan Arcs, respectively. The Tadamigawa older-stage granites are located to the west of the Tanakura Tectonic Line (TTL), which is the boundary of the Northeastern Japan Arcs and the Ashio belt, and the formation age and source of the Tadamigawa older-stage granites were unclear. In this study, we determined the formation age of the Tadamigwa older-stage granites by zircon U-Pb and whole-rock and mineral $\mathrm{Rb}$-Sr datings and estimated the origin of the granites by the initial $\mathrm{Sr}$ isotopic ratio. The Tadamigawa older-stage granites have two intrusion ages, 107-93 Ma and ca. $62 \mathrm{Ma}$. In addition, the older granites show $+4.6 \varepsilon_{\mathrm{Sr}}-+20.4 \varepsilon_{\mathrm{Sr}}$, and the younger granites show $+64.0 \varepsilon_{\mathrm{Sr}}$. The Tadamigawa older-stage granites to the west of the TTL of northeastern Japan comprise two types of plutons with different ages and origins.
\end{abstract}

Keywords: granite, zircon U-Pb age, Rb-Sr age, Northeastern Japan Arc, Cretaceous to Paleogene

\section{INTRODUCTION}

The Cretaceous to early Paleogene magmatism in the Honshu Arc was characterized by successive geographical transitions of the magmatic activities both in the Southwestern and Northeastern Japan Arcs, respectively (e.g., Kagami et al., 1992, 1999; Kagami, 2005). In the Southwestern Japan Arc, the ages of the igneous rocks vary from $100 \mathrm{Ma}$ in the western part to $65 \mathrm{Ma}$ in the eastern part, and the magmatism was continuous from $100 \mathrm{Ma}$ to $65 \mathrm{Ma}$. In the Northeastern Japan Arc (including the Ashio belt), the ages of the igneous rocks vary from $130 \mathrm{Ma}$ in the northeastern part to $60 \mathrm{Ma}$ in the southwestern part, and the magmatism occurred in two separate periods, 110 $100 \mathrm{Ma}$ and 75-60 Ma. The Southwestern Japan Arc magmatism occurred along the Median Tectonic Line (MTL), which is an important geological boundary within

\footnotetext{
*Corresponding author (e-mail: wakasugi@kwansei.ac.jp)

Copyright $\odot 2020$ by The Geochemical Society of Japan.
}

southwestern Japan. The magmatism of the Northeastern Japan Arc, on the other hand, is not related to tectonic lines, such as the MTL. The area to the west of the Tanakura Tectonic Line (TTL, Kinoshita and Ito, 1990; Nakajima et al., 1990) is the boundary between the Northeastern Japan Arc and the Ashio belt and the TTL is related to the formation of the Sea of Japan (e.g., Otsuki and Ehiro, 1978; Sato and Amano, 1991). In the area to the west of the TTL, late Cretaceous to early Paleogene granites widely intrude into the Jurassic accretionary complex (Ashio belt) (Takahashi et al., 2005) (Fig. 1). Several researchers (e.g., Kawano and Ueda, 1966; Tanioka et al., 2014) have suggested the possibility that the formation ages and magma sources of the granites in the western side of the TTL are different from the typical magmatism in the Northeastern Japan Arc. Geochemical and chronological studies of the granites in the western side of the TTL can provide constraints on the formation history of igneous rocks in the Northeastern Japan Arc.

The Tadamigawa older-stage granites are distributed in the Okutadami area located west of the TTL with late 


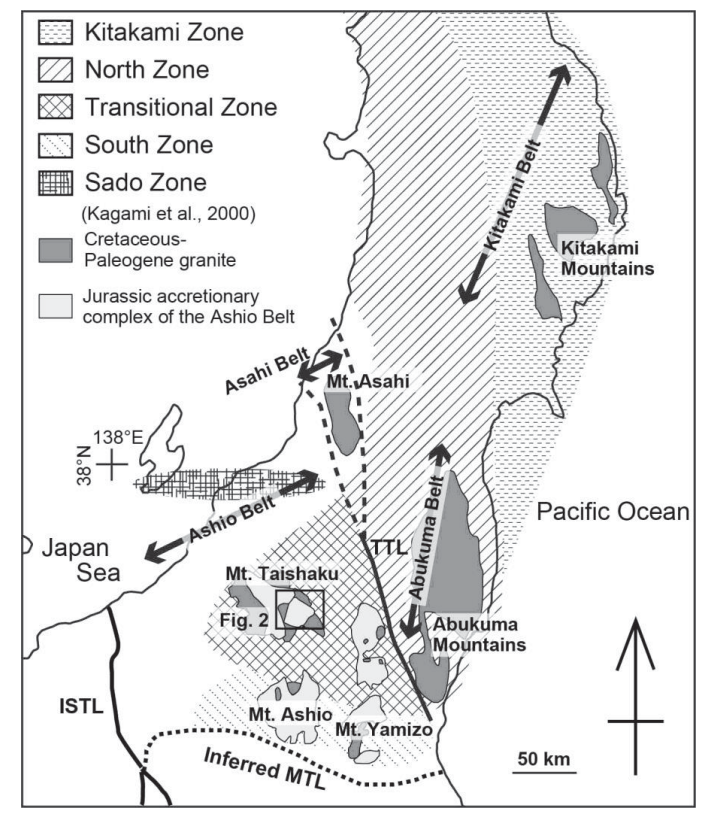

Fig. 1. Distribution of Cretaceous to Paleogene granitoids in the Northeastern Japan Arc. Isotopic zones (Kitakami, North, Transitional, South, and Sado Zones) are from Kagami et al. (2000). TTL: Tanakura Tectonic Line, MTL: Median Tectonic Line, ISTL: Itoigawa-Shizuoka Tectonic Line.

Cretaceous to early Paleogene formation ages. The Tadamigawa older-stage granites occur mainly in three areas: the Taishaku Mountains, the Tadamigawa and the Inagawa areas (Fig. 2). These granite plutons have been interpreted as generated from the same parental magma based on their similarity of the major and trace element compositions (Tanioka et al., 2014). However, the K-Ar biotite ages of these plutons vary from $100 \mathrm{Ma}$ to $60 \mathrm{Ma}$ (Kawano and Ueda, 1966; Tanioka et al., 2014). Tanioka et al. (2014) proposed two possibilities to explain this age discrepancy: 1) the younger K-Ar age corresponds to the secondary resetting of the K-Ar system by thermal effects of later magmatism, or 2) the magmatic activity and the source magma are different for each pluton. However, no clear and detailed interpretation has been made for this age discrepancy yet. A chronological study using multiple radiogenic isotope systems will be key to understanding the formation ages for the Tadamigawa olderstage granites. Whole-rock and mineral $\mathrm{Rb}-\mathrm{Sr}$ isochron methods yield ages with initial $\mathrm{Sr}$ isotope ratios (SrI), which can be used to estimate the magmatic origin of the pluton or cooling ages with corresponding $\mathrm{Sr}$ isotope ratios, which may not necessarily allow the estimation of the magmatic origin of the pluton. In addition, zircon U$\mathrm{Pb}$ dating provided the crystallization ages of igneous rocks, and its closure temperature $\left(900^{\circ} \mathrm{C}\right.$ : Cherniak and Watson, 2001) is significantly higher than those found

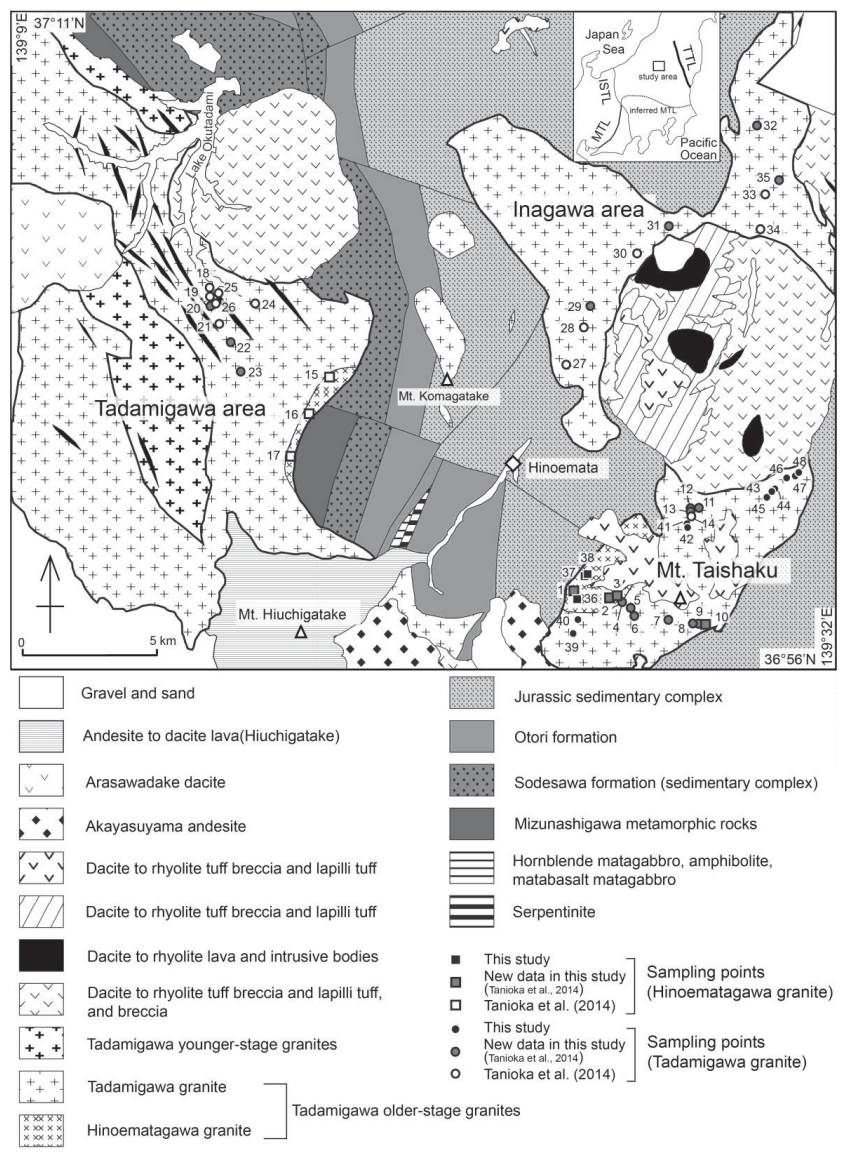

Fig. 2. Geological map of the Okutadami area (Yamamoto et al., 2000; Tanioka et al., 2014). Sampling points of the Hinoematagawa and the Tadamigawa granites are shown as squares and circles, respectively. TTL: Tanakura Tectonic Line, MTL: Median Tectonic Line, ISTL: Itoigawa-Shizuoka Tectonic Line.

via the other dating methods, such as $\mathrm{Rb}-\mathrm{Sr}$ (biotite: $310^{\circ} \mathrm{C}$; K-felspar: $360^{\circ} \mathrm{C}$, Nishimura and Mogi, 1986) and $\mathrm{K}-\mathrm{Ar}\left(300^{\circ} \mathrm{C}\right.$, Verschure et al., 1980) systems. Here, we report the zircon $\mathrm{U}-\mathrm{Pb}$ and whole-rock and mineral $\mathrm{Rb}$ $\mathrm{Sr}$ ages of the Tadamigawa older-stage granites to discuss the formation history of these granitic rocks. In addition, we discuss the magmatic origins of each pluton given the initial $\mathrm{Sr}$ isotopic ratio (SrI). A detailed understanding of the formation age and magma source of the Tadamigawa older-stage granites will add further insights into the magmatic history of the Northeastern Japan Arc.

\section{Geological Background and Petrography}

The Cretaceous to early Paleogene magmatism in northeastern Japan formed intermittent igneous plutons, such as the Matsuki granite (116 Ma; Kawano and Ueda, 1966), the Sori granite (93 Ma; Sudo et al., 1998) and the 


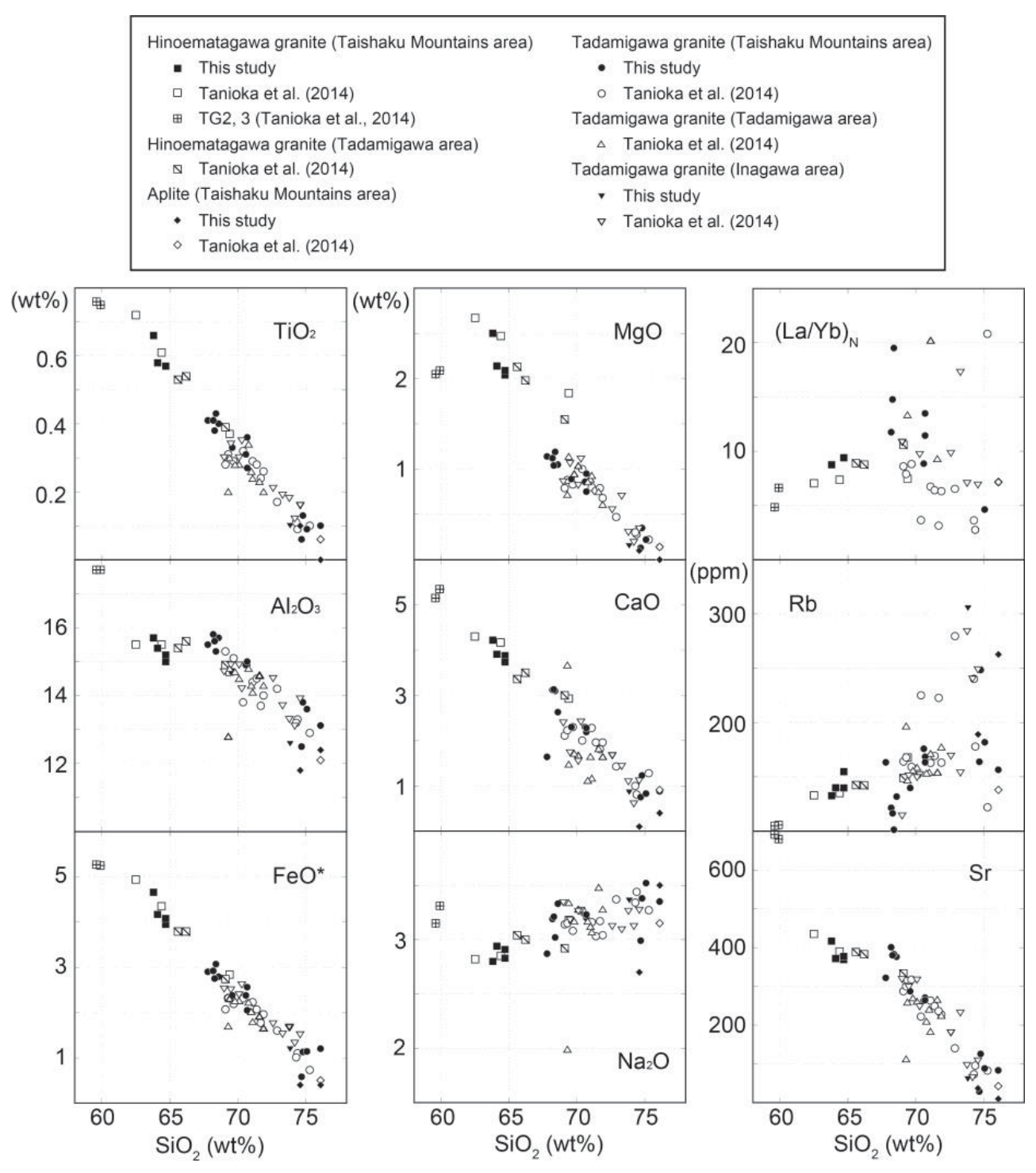

Fig. 3. Harker's diagram for selected major and trace elements of the Tadamigawa older-stage granites. A part of the data from Tanioka et al. (2014).

Chuzenji acidic volcanic rocks (70-60 Ma; Kawano and Ueda, 1966) in the Ashio Mountains area (Yanai, 1972, 1973a, 1973b) (Fig. 1). The Tadamigawa older-stage and younger-stage granites in the Okutadami area are distributed along with these successive granite plutons. In the northern part of this region, the ages of the granite plutons tend to become successively younger from northeast to southwest: the Kitakami granites (120-110 Ma: e.g., Shibata and Miller, 1962; Kawano and Ueda, 1965a; Nozawa, 1975), plutonic rocks of the Abukuma area (10090 Ma: e.g., Kawano and Ueda, 1965b; Ueno, 1977; Shibata and Utsumi, 1983) and the Asahi and Ashio granites (90-60 Ma: e.g., Kawano and Ueda, 1966; Shibata, 1966) (Fig. 1). On the east side of the Okutadami area, both the older pluton (ca. 105 Ma: Shibata et al., 1973) and the younger pluton ( $c a .65 \mathrm{Ma}$ : Kawano and Ueda, 1966) are distributed in the Yamizo Mountains area (Fig. 1).

The geology of the Okutadami area is similar to that of the Ashio Mountains area, which is located next to the south of the Okutadami area. Both the Okutadami and Ashio Mountains areas consist mainly of Paleozoic to Mesozoic Ashio accretionary complex, Cretaceous to early Paleogene granites and acidic volcanic rocks, and Neogene to Quaternary volcanic rocks (Fig. 2). The Ashio accretionary complex consists of mudstone, sandstone and chert with basalt and limestone (Noda et al., 2014). The ages of the Tadamigawa older-stage granites are estimated 
as late Cretaceous to early Paleogene (Chihara and Komatsu, 1992; Yamamoto et al., 2000). The late Paleogene Tadamigawa younger-stage granites are distributed around the older-stage rocks. The distribution areas of the Tadamigawa older-stage granite outcrops are restricted by a cover of Neogene to Quaternary volcanic rocks.

In this study area, the Tadamigawa older-stage granites are distributed mainly in the Taishaku Mountains, the Tadamigawa and the Inagawa areas (Fig. 2). The Tadamigawa older-stage granites can be divided into two rock types: the Hinoematagawa granite and the Tadamigawa granite. The Hinoematagawa granite is distributed in the southwestern part of the Taishaku Mountains area and also in the eastern part of the Tadamigawa area in a small section, both of which are enclosed by the Tadamigawa granite. The Tadamigawa granite is distributed in the Taishaku Mountains, the Tadamigawa and the Inagawa areas. (In Fig. 3, the Hinoematagawa and Tadamigawa granite samples are distinguished by different symbols.)

The Hinoematagawa granite is quartz monzonite to granite, medium-grained and consists mainly of quartz, plagioclase, K-feldspar and biotite. The accessory minerals are zircon, apatite and opaque minerals. The Tadamigawa granite is granite to granodiorite, coarsegrained, and consists mainly of quartz, plagioclase, Kfeldspar, biotite and hornblende. The accessory minerals are zircon, apatite and opaque minerals (Tanioka et al., 2014). Aplite intrudes into the Tadamigawa granite in some parts of the Taishaku Mountains area and consists mainly of quartz, plagioclase, K-feldspar and biotite. Neogene Tashiroyama rhyolite intrudes into the Tadamigawa granite in the Taishaku Mountains area (Murayama and Kawata, 1955; Yamamoto et al., 2000). Many late Cretaceous basic-felsic dykes intrude into the Tadamigawa older-stage granites in the Tadamigawa area. The Hinoematagawa granite has suffered contact metamorphism from the Tadamigawa granite at the boundary between the two plutons (Murayama and Kawata, 1955). This observation clearly shows that the Tadamigawa granite is younger than the Hinoematagawa granite. Kawano and Ueda (1966) reported a K-Ar biotite age of $69 \mathrm{Ma}$ (recalculated by the decay constant of Steiger and Jäger, 1977) for the Tadamigawa granite in the southern part of the Inagawa area. Tanioka et al. (2014) reported K-Ar biotite ages of $102.3 \pm 2.2 \mathrm{Ma}$ and $102.7 \pm 2.2 \mathrm{Ma}$ for the Hinoematagawa and Tadamigawa granites in the Taishaku Mountains area, respectively, as well as K-Ar biotite ages of $89.6 \pm 1.9 \mathrm{Ma}$ and $61.7 \pm 1.4 \mathrm{Ma}$ for the Tadamigawa granite in the Tadamigawa and Inagawa areas, respectively.

In this study, twenty samples from the three areas (TG9c, d, e, 29b, 36-48: Fig. 2) were prepared for chemi- cal analysis, zircon U-Pb dating and whole-rock and/or mineral $\mathrm{Rb}$-Sr isochron. Some of the samples analyzed for zircon $\mathrm{U}-\mathrm{Pb}$ dating and whole-rock and/or mineral $\mathrm{Rb}$ $\mathrm{Sr}$ isochron were from Tanioka et al. (2014). Eight samples (TG9b, 20, 22, 23, 29a, 31, 32, and 35) were analyzed for REEs by ICP-MS although they were already measured for major and trace elements by XRF in Tanioka $e t$ al. (2014). The chemical composition data of the samples TG1 - 35 from Tanioka et al. (2014) were also used with the new chemical data set for discussion.

\section{Analytical Technique}

\section{Major and trace elements}

The concentrations of 10 major and 15 trace elements were measured by an X-ray fluorescence spectrometer (Shimadzu XRF-1800) with an Rh target using the glass bead method at Kwansei Gakuin University. The rock powder samples and flux $\left(\mathrm{Li}_{2} \mathrm{~B}_{4} \mathrm{O}_{7}\right)$ were mixed in proportions of 0.7:6.0 g for major elements and 2.0:3.0 $\mathrm{g}$ for trace elements and were melted to prepare glass beads. The tube voltage and current were set at $40 \mathrm{kV}$ and 70 $\mathrm{mA}$ for major elements and at $40 \mathrm{kV}$ and $95 \mathrm{~mA}$ for trace elements. The analytical method was based on that of Nakazaki et al. (2004). The loss on ignition (LOI) was measured by simple weighing after heating of $500 \mathrm{mg}$ of rock powder samples at $1000^{\circ} \mathrm{C}$ for 2 hours.

The concentrations of rare earth elements (REE; except for $\mathrm{Sc}$ ) were measured by an internal standard method using In with an inductively coupled plasma mass spectrometer (ICP-MS) (Agilent Technologies 7500cx) at Kwansei Gakuin University. The sample for the REE analysis was prepared from the XRF glass bead, a part of which was dissolved with nitric acid. The REE were separated from the dissolved sample by cation-exchange resin (Bio-Rad AG50W-X8).

\section{Rb-Sr system}

Mineral samples (plagioclase, K-feldspar, biotite) were separated from the whole-rock powder by magnetic separation and heavy liquid (sodium polytungstate, SPT) techniques. Approximately $40 \mathrm{mg}$ of the rock or mineral powder samples were decomposed with a mixture of $\mathrm{HF}$, $\mathrm{HNO}_{3}$ and $\mathrm{HClO}_{4}$. A decomposed sample was split into several aliquots for isotopic and quantitative analysis. An aliquot of a decomposed sample was used for the $\mathrm{Rb}$ and $\mathrm{Sr}$ concentration analysis. Strontium was separated from the aliquot by extraction chromatography using $\mathrm{Sr} \mathrm{Spec}$ resin (Eichrom Technologies Inc.).

The $\mathrm{Sr}$ isotope ratios were measured with a thermal ionization mass spectrometer (TIMS) (Thermo Finnigan TRITON) at the Kochi Core Center (KCC), Japan. The $\mathrm{Sr}$ fraction was loaded on a single $\mathrm{W}$ filament with a $\mathrm{Ta}$ activator and $\mathrm{HNO}_{3}$. The mass fractionation during the 
Table 1. Rb and Sr concentration measured by isotope dilution and ICP-MS methods

\begin{tabular}{|c|c|c|c|c|c|c|c|c|c|}
\hline \multirow[t]{2}{*}{ Sample } & \multirow{2}{*}{$\begin{array}{l}\text { Sample } \\
(\mathrm{mg})\end{array}$} & \multirow{2}{*}{$\begin{array}{c}\mathrm{Rb}(\mathrm{ppm}) \\
\mathrm{ID} *\end{array}$} & \multirow{2}{*}{$\begin{array}{l}\mathrm{Sr}(\mathrm{ppm}) \\
\text { ID* }\end{array}$} & \multirow{2}{*}{$\begin{array}{l}\mathrm{Rb}(\mathrm{ppm}) \\
\mathrm{ICP}\end{array}$} & \multirow{2}{*}{$\begin{array}{l}\mathrm{Sr}(\mathrm{ppm}) \\
\mathrm{ICP}\end{array}$} & \multicolumn{2}{|c|}{${ }^{87} \mathrm{Rb} /{ }^{86} \mathrm{Sr}$} & & \multirow{2}{*}{$\begin{array}{c}\Delta^{87} \mathrm{Rb} /{ }^{8}{ }^{6} \mathrm{Sr}(\%) \\
\text { (ID-ICP) }\end{array}$} \\
\hline & & & & & & ID* & ICP & & \\
\hline JB-3 & 25.15 & $15.0(1)$ & $409(3)$ & 13.7 & 386 & $0.106(1)$ & 0.103 & & 2.8 \\
\hline JB-3 & 66.97 & $15.1(1)$ & $415(3)$ & 13.7 & 384 & 0.105 (1) & 0.103 & & 1.9 \\
\hline JB-3 & 97.21 & $14.9(1)$ & $411(3)$ & 13.7 & 390 & 0.105 (1) & 0.102 & & 2.9 \\
\hline JA-2 & 52.32 & $70.02(7)$ & $244(2)$ & 68.6 & 235 & $0.83(1)$ & 0.844 & & 1.7 \\
\hline JG-1a & 109.64 & $173(1)$ & $181(1)$ & 173 & 179 & $2.77(3)$ & 2.80 & & 1.1 \\
\hline JR-1 & 123.57 & $244(2)$ & $27.7(2)$ & 248 & 28.3 & $25.5(2)$ & 25.3 & & 0.8 \\
\hline JR-2 & 83.02 & $291(2)$ & $7.94(6)$ & 296 & 7.94 & $106(1)$ & 108 & & 1.9 \\
\hline JB-1a & 77.01 & $36.8(3)$ & $444(3)$ & 38.1 & 439 & $0.240(2)$ & 0.251 & & 4.6 \\
\hline JA-3 & 53.88 & $35.3(2)$ & $285(2)$ & 35.1 & 280 & $0.359(3)$ & 0.363 & & 1.1 \\
\hline \multirow[t]{2}{*}{ BCR-2 } & 87.47 & $47.8(3)$ & $337(2)$ & 46.9 & 336 & $0.410(4)$ & 0.404 & & 1.5 \\
\hline & & & & & & & & Average (2SD) & $2 \pm 2$ \\
\hline
\end{tabular}

*The number in parenthesis indicates the 2SD analytical error on the last figure.

measurement was corrected by internal normalization using exponential law and ${ }^{86} \mathrm{Sr} /{ }^{88} \mathrm{Sr}=0.1194$. The detail measurement conditions are described in Wakaki et al. (2017). Repeated analyses of NIST SRM 987 during this study yielded ${ }^{87} \mathrm{Sr} /{ }^{86} \mathrm{Sr}$ ratios of $0.710243 \pm 5(2 \mathrm{SD}, \mathrm{n}=$ 7). The measured ${ }^{87} \mathrm{Sr} /{ }^{86} \mathrm{Sr}$ ratio of the sample was further corrected to ${ }^{87} \mathrm{Sr} /{ }^{86} \mathrm{Sr}_{\text {SRM- } 987}=0.710248$ to account for the small possible interlaboratory bias. The concentrations of $\mathrm{Rb}$ and $\mathrm{Sr}$ were measured with ICP-MS (Agilent Technologies 7700) at the KCC using In internal standardization method, and the ${ }^{87} \mathrm{Rb} /{ }^{86} \mathrm{Sr}$ ratio was calculated from these concentration data.

To estimate the accuracy of the ${ }^{87} \mathrm{Rb} /{ }^{86} \mathrm{Sr}$ ratio measured by ICP-MS, we analyzed the $\mathrm{Rb}$ and $\mathrm{Sr}$ concentrations of eight reference rocks issued from the Geological Survey of Japan (GSJ) and the USGS using the isotope dilution (ID) method together with ICP-MS and compared the results. An aliquot of a decomposed reference rock sample was spiked with ${ }^{84} \mathrm{Sr}$ and ${ }^{85} \mathrm{Rb}$ at Nagoya University (Wakaki and Tanaka, 2005). The spiked $\mathrm{Rb}$ and $\mathrm{Sr}$ were then separated from the matrix elements with cationexchange resin (Bio-Rad AG50W-X8). The isotope ratios of $\mathrm{Rb}$ and $\mathrm{Sr}\left({ }^{85} \mathrm{Rb} /{ }^{87} \mathrm{Rb}\right.$ and $\left.{ }^{84} \mathrm{Sr} /{ }^{86} \mathrm{Sr}\right)$ for the spiked samples were measured with Sector 54-30 TIMS at Nagoya University, Japan, and the ${ }^{87} \mathrm{Rb} /{ }^{86} \mathrm{Sr}$ ratio was calculated from the $\mathrm{Rb}$ and $\mathrm{Sr}$ concentration data. The other aliquot was also analyzed for the ${ }^{87} \mathrm{Rb} /{ }^{86} \mathrm{Sr}$ ratio using ICP-MS. The results are summarized in Table 1. The deviation of the ${ }^{87} \mathrm{Rb} /{ }^{86} \mathrm{Sr}$ ratio analyzed by ICP-MS from that analyzed by ID was expressed as follows:

$$
\begin{aligned}
& \Delta^{87} \mathrm{Rb} /{ }^{86} \mathrm{Sr}(\%) \\
& =\left|\left({ }^{87} \mathrm{Rb} /{ }^{86} \mathrm{Sr}\right)_{\mathrm{ICP}-\mathrm{MS}} /\left({ }^{87} \mathrm{Rb} /{ }^{86} \mathrm{Sr}\right)_{\mathrm{ID}}-1\right| \times 100 .
\end{aligned}
$$

The $\Delta^{87} \mathrm{Rb} /{ }^{86} \mathrm{Sr}$ value ranged from 0.8 to 4.6 with an average of $2 \pm 2$. Based on the reliability of the ID data, we took the upper end of the 2SD range and estimated the accuracy of our ${ }^{87} \mathrm{Rb} /{ }^{86} \mathrm{Sr}$ data measured by ICP-MS as $4 \%$.

The $\mathrm{Rb}$ and $\mathrm{Sr}$ contents and ${ }^{87} \mathrm{Sr} /{ }^{86} \mathrm{Sr}$ ratio for the GSJ (Geological Survey of Japan) reference rock JG-2 were also analyzed as follows: ${ }^{87} \mathrm{Sr} /{ }^{86} \mathrm{Sr}=0.759876 \pm 3$ (2SD), $\mathrm{Rb}=321 \mathrm{ppm}$ and $\mathrm{Sr}=17.6 \mathrm{ppm}$.

\section{$U$-Pb system}

The $\mathrm{U}$ and $\mathrm{Pb}$ isotope ratios in zircon were measured by an inductively coupled plasma mass spectrometer (ICP-MS, Agilent Technologies 7700x) using the laser ablation (LA) system (Electro Scientific Industries NWR213) at Nagoya University. Zircon grains were concentrated from samples pulverized to $<250 \mu \mathrm{m}$ through a heavy liquid (SPT) technique. Approximately 50 zircon grains for each sample were collected from the heavier mineral fractions under a binocular microscope by hand picking. Back-scattered electron (BSE) and a cathodoluminescence images (CL) were taken using a scanning electron microscope (SEM) (Hitachi S-3400N at Nagoya University Museum) (JEOL JSM-6500F at $\mathrm{KCC}$ ) equipped with cathodoluminescence system to check the internal structure and morphology of the zircon grains as a guide to selecting good sites for $\mathrm{U}-\mathrm{Pb}$ measurement. The U-Pb analysis of zircon grains by LAICP-MS was performed using a 91500 zircon standard (1062.4 Ma; Wiedenbeck et al., 1995) and a NIST SRM 610 glass standard (Horn and Blanckenburg, 2007). The diameter of the laser spot was $25 \mu \mathrm{m}$. Detailed analytical methods are described in Orihashi et al. (2008) and Kouchi et al. (2015).

\section{RESUlTS}

\section{Major and trace elements}

The major and trace element concentrations of the Tadamigawa older-stage granites are shown in Table 2 
Table 2. Whole-rock chemical compositions of the Tadamigawa older-stage granites

\begin{tabular}{|c|c|c|c|c|c|c|c|c|c|c|c|c|c|c|}
\hline & \multicolumn{14}{|c|}{ Taishaku Mountains area } \\
\hline & \multicolumn{4}{|c|}{ Hinoematagawa granite } & \multicolumn{10}{|c|}{ Tadamigawa granite } \\
\hline & TG36 & TG37a & TG37b & TG38 & TG9b & TG9e & TG39a & TG39b & TG40 & TG41 & TG42 & TG43 & TG44 & TG45 \\
\hline \multicolumn{15}{|l|}{$(\mathrm{wt} \%)$} \\
\hline $\mathrm{SiO}_{2}$ & 63.8 & 64.1 & 64.7 & 64.7 & $75.33^{\mathrm{a}}$ & 76.1 & 70.7 & 70.6 & 74.7 & 74.8 & 75.1 & 70.7 & 68.6 & 69.6 \\
\hline $\mathrm{TiO}_{2}$ & 0.66 & 0.58 & 0.57 & 0.57 & $0.10^{\mathrm{a}}$ & 0.10 & 0.27 & 0.31 & 0.06 & 0.13 & 0.09 & 0.36 & 0.40 & 0.33 \\
\hline $\mathrm{Al}_{2} \mathrm{O}_{3}$ & 15.68 & 15.41 & 15.21 & 15.02 & $12.86^{\mathrm{a}}$ & 13.12 & 14.92 & 14.86 & 12.52 & 13.78 & 13.62 & 14.99 & 15.72 & 14.66 \\
\hline $\mathrm{FeO}^{*}$ & 4.66 & 4.17 & 3.95 & 4.08 & $0.73^{\mathrm{a}}$ & 1.20 & 2.05 & 2.38 & 0.58 & 1.13 & 1.14 & 2.56 & 2.79 & 2.38 \\
\hline $\mathrm{MnO}$ & 0.12 & 0.10 & 0.10 & 0.10 & $0.03^{\mathrm{a}}$ & 0.04 & 0.07 & 0.09 & 0.01 & 0.08 & 0.05 & 0.07 & 0.08 & 0.06 \\
\hline $\mathrm{MgO}$ & 2.50 & 2.14 & 2.04 & 2.09 & $0.22^{\mathrm{a}}$ & 0.14 & 0.75 & 0.86 & 0.13 & 0.35 & 0.22 & 0.95 & 1.05 & 0.89 \\
\hline $\mathrm{CaO}$ & 4.22 & 3.91 & 3.88 & 3.74 & $1.28^{\mathrm{a}}$ & 0.88 & 2.19 & 2.30 & 0.75 & 1.23 & 0.83 & 2.28 & 2.63 & 2.30 \\
\hline $\mathrm{Na}_{2} \mathrm{O}$ & 2.80 & 2.94 & 2.91 & 2.83 & $3.27^{\mathrm{a}}$ & 3.35 & 3.20 & 3.23 & 2.99 & 3.38 & 3.52 & 3.23 & 3.33 & 3.17 \\
\hline $\mathrm{K}_{2} \mathrm{O}$ & 3.82 & 3.92 & 3.86 & 3.87 & $4.21^{\mathrm{a}}$ & 4.76 & 4.63 & 4.25 & 4.91 & 4.41 & 4.48 & 4.21 & 3.71 & 3.79 \\
\hline $\mathrm{P}_{2} \mathrm{O}_{5}$ & 0.21 & 0.19 & 0.19 & 0.19 & $0.02^{\mathrm{a}}$ & 0.02 & 0.09 & 0.10 & 0.01 & 0.04 & 0.03 & 0.12 & 0.13 & 0.11 \\
\hline LOI & 1.02 & 0.64 & 0.59 & 0.76 & $0.38^{\mathrm{a}}$ & 0.46 & 0.41 & 0.30 & 0.36 & 0.38 & 0.77 & 0.64 & 1.27 & 0.54 \\
\hline Total & 99.5 & 98.1 & 98.0 & 98.0 & $98.4^{\mathrm{a}}$ & 100.2 & 99.3 & 99.2 & 97.0 & 99.7 & 99.8 & 100.1 & 99.7 & 97.8 \\
\hline \multicolumn{15}{|l|}{ (ppm) } \\
\hline V & 105 & 92.6 & 88.3 & 92.0 & $18.9^{\mathrm{a}}$ & 15.8 & 36.2 & 41.0 & 15.6 & 21.3 & 19.7 & 43.9 & 52.3 & 42.9 \\
\hline $\mathrm{Cr}$ & 33.5 & 29.6 & 26.1 & 30.7 & $1.3^{\mathrm{a}}$ & 2.0 & 6.8 & 8.6 & 0.9 & 2.3 & 3.8 & 10.2 & 8.2 & 10.2 \\
\hline Co & 19.5 & 16.1 & 16.4 & 17.2 & $2.1^{\mathrm{a}}$ & 3.4 & 6.5 & 7.8 & 2.3 & 4.6 & 3.5 & 9.2 & 8.8 & 7.8 \\
\hline $\mathrm{Ni}$ & 5.4 & 7.1 & 7.2 & 5.0 & $4.2^{\mathrm{a}}$ & 4.3 & 4.6 & 4.5 & 4.9 & 5.4 & 5.0 & 5.4 & 4.6 & 5.7 \\
\hline $\mathrm{Cu}$ & 1.4 & 4.5 & 1.2 & 2.7 & $0.3^{\mathrm{a}}$ & n.d. & 0.6 & n.d. & 0.3 & n.d. & n.d. & 0.9 & 5.7 & n.d. \\
\hline $\mathrm{Zn}$ & 63.1 & 57.3 & 57.7 & 56.1 & $17.4^{\mathrm{a}}$ & 19.5 & 39.3 & 44.2 & 7.5 & 35.4 & 25.1 & 46.9 & 47.0 & 44.5 \\
\hline $\mathrm{Rb}$ & 133 & 140 & 155 & 140 & $122^{\mathrm{a}}$ & 156 & 168 & 176 & 164 & 248 & 182 & 163 & 132 & 140 \\
\hline $\mathrm{Sr}$ & 417 & 372 & 369 & 377 & $82.5^{\mathrm{a}}$ & 82.8 & 270 & 263 & 28.3 & 126 & 87.7 & 264 & 376 & 287 \\
\hline Y & 23.1 & 22.6 & 22.1 & 22.2 & $20.7^{\mathrm{a}}$ & 18.5 & 20.4 & 23.8 & 22.4 & 27.8 & 33.7 & 22.4 & 21.8 & 20.8 \\
\hline $\mathrm{Zr}$ & 161 & 137 & 142 & 142 & $84.6^{\mathrm{a}}$ & 115 & 120 & 137 & 55.8 & 80.8 & 78.1 & 142 & 170 & 137 \\
\hline $\mathrm{Nb}$ & 5.1 & 4.7 & 5.1 & 4.2 & $6.0^{\mathrm{a}}$ & 2.3 & 3.5 & 5.5 & 2.2 & 4.3 & 4.7 & 5.0 & 5.1 & 4.7 \\
\hline $\mathrm{Ba}$ & 476 & 412 & 413 & 416 & $182^{\mathrm{a}}$ & 160 & 491 & 470 & 53.6 & 265 & 383 & 374 & 545 & 413 \\
\hline $\mathrm{Pb}$ & 17.6 & 16.0 & 18.6 & 18.2 & $20.6^{\mathrm{a}}$ & 26.0 & 24.5 & 26.1 & 30.5 & 32.7 & 29.4 & 20.8 & 18.4 & 21.3 \\
\hline Th & 13.7 & 13.2 & 12.9 & 15.9 & $16.8^{\mathrm{a}}$ & 19.8 & 12.2 & 15.2 & 22.4 & 24.7 & 9.5 & 14.5 & 11.8 & 23.3 \\
\hline Y & 18.4 & & & 17.4 & 13.3 & & 12.9 & 16.4 & & & 22.9 & 14.8 & & \\
\hline $\mathrm{La}$ & 22.7 & & & 23.7 & 40.2 & & 25.8 & 21.2 & & & 15.2 & 22.7 & & \\
\hline $\mathrm{Ce}$ & 45.9 & & & 47.2 & 72.8 & & 47.7 & 44.1 & & & 30.7 & 42.5 & & \\
\hline $\operatorname{Pr}$ & 5.16 & & & 5.15 & 8.09 & & 5.13 & 4.41 & & & 3.56 & 4.81 & & \\
\hline $\mathrm{Nd}$ & 19.5 & & & 18.8 & 27.5 & & 17.4 & 15.3 & & & 12.9 & 17.2 & & \\
\hline $\mathrm{Sm}$ & 3.88 & & & 3.68 & 4.34 & & 2.96 & 2.99 & & & 3.09 & 3.24 & & \\
\hline $\mathrm{Eu}$ & 0.99 & & & 0.98 & 0.58 & & 0.76 & 0.73 & & & 0.33 & 0.73 & & \\
\hline $\mathrm{Gd}$ & 3.25 & & & 3.10 & 3.42 & & 2.54 & 2.63 & & & 2.81 & 2.79 & & \\
\hline $\mathrm{Tb}$ & 0.51 & & & 0.47 & 0.40 & & 0.37 & 0.42 & & & 0.54 & 0.42 & & \\
\hline Dy & 3.00 & & & 2.89 & 2.15 & & 2.10 & 2.62 & & & 3.49 & 2.49 & & \\
\hline Ho & 0.61 & & & 0.58 & 0.44 & & 0.41 & 0.52 & & & 0.70 & 0.49 & & \\
\hline Er & 1.76 & & & 1.69 & 1.39 & & 1.23 & 1.60 & & & 2.12 & 1.45 & & \\
\hline $\mathrm{Tm}$ & 0.26 & & & 0.27 & 0.23 & & 0.19 & 0.25 & & & 0.32 & 0.21 & & \\
\hline $\mathrm{Yb}$ & 1.79 & & & 1.74 & 1.34 & & 1.32 & 1.66 & & & 2.28 & 1.38 & & \\
\hline $\mathrm{Lu}$ & 0.26 & & & 0.28 & 0.24 & & 0.20 & 0.26 & & & 0.31 & 0.21 & & \\
\hline $\mathrm{La}_{\mathrm{N}} / \mathrm{Sm}_{\mathrm{N}}$ & 3.66 & & & 4.03 & 5.81 & & 5.46 & 4.45 & & & 3.09 & 4.39 & & \\
\hline $\mathrm{Gd}_{\mathrm{N}} / \mathrm{Yb}_{\mathrm{N}}$ & 1.50 & & & 1.47 & 2.12 & & 1.59 & 1.31 & & & 1.02 & 1.68 & & \\
\hline $\mathrm{La}_{\mathrm{N}} / \mathrm{Yb}_{\mathrm{N}}$ & 8.76 & & & 9.40 & 20.8 & & 13.5 & 8.86 & & & 4.62 & 11.4 & & \\
\hline $\mathrm{Eu} / \mathrm{Eu}^{\#}$ & 0.85 & & & 0.88 & 0.46 & & 0.84 & 0.79 & & & 0.34 & 0.74 & & \\
\hline$\sum$ REE & 110 & & & 111 & 163 & & 108 & 99 & & & 78 & 101 & & \\
\hline
\end{tabular}

and Fig. 3. Data from Tanioka et al. (2014) are included in Fig. 3. The $\mathrm{SiO}_{2}$ contents range from 63.8 to $64.7 \mathrm{wt} \%$ for the Hinoematagawa granite, from 67.8 to $76.1 \mathrm{wt} \%$ for the Tadamigawa granite and from 74.6 to $76.1 \mathrm{wt} \%$ for the aplite in the Tadamigawa granite in this study. The concentrations of $\mathrm{K}_{2} \mathrm{O}$, Ni and Th increased with the $\mathrm{SiO}_{2}$ content, and those of $\mathrm{TiO}_{2}, \mathrm{Al}_{2} \mathrm{O}_{3}, \mathrm{FeO} * \mathrm{MnO}, \mathrm{MgO}$, $\mathrm{CaO}, \mathrm{P}_{2} \mathrm{O}_{5}, \mathrm{~V}, \mathrm{Cr}, \mathrm{Co}, \mathrm{Cu}, \mathrm{Zn}$ and $\mathrm{Ba}$ all decreased with the $\mathrm{SiO}_{2}$ content. The $\mathrm{Na}_{2} \mathrm{O}$ contents remained relatively constant regardless of the $\mathrm{SiO}_{2}$ increase. The concentrations of $\mathrm{Rb}, \mathrm{Y}, \mathrm{Zr}, \mathrm{Nb}$ and $\mathrm{Pb}$ were scattered for the $\mathrm{SiO}_{2}{ }^{-}$ rich samples. The copper $(\mathrm{Cu})$ contents were mostly less than the detection limit of the XRF analysis. These results are consistent with the results from Tanioka et al. (2014). Two samples (TG2, 3) of the Hinoematagawa granite in the Taishaku Mountains area showed a different trend for $\mathrm{Al}_{2} \mathrm{O}_{3}, \mathrm{MgO}$ and $\mathrm{Sr}$ from the other Hinoematagawa granites. 
Table 2. (continued)

\begin{tabular}{|c|c|c|c|c|c|c|c|c|c|c|c|c|c|c|}
\hline & \multicolumn{9}{|c|}{ Taishaku Mountains area } & \multicolumn{5}{|c|}{ Inagawa area } \\
\hline & \multicolumn{4}{|c|}{ Tadamigawa granite } & \multicolumn{2}{|c|}{ Aplite } & \multicolumn{3}{|c|}{ Tadamigawa granite } & \multicolumn{5}{|c|}{ Tadamigawa granite } \\
\hline & TG46a & TG46b & TG47 & TG48 & TG9c & TG9d & TG20 & TG22 & TG23 & TG29a & TG29b & TG31 & TG32 & TG35 \\
\hline$(\mathrm{wt} \%)$ & & & & & & & & & & & & & & \\
\hline $\mathrm{SiO}_{2}$ & 68.4 & 67.8 & 68.2 & 68.3 & 76.1 & 74.6 & $71.6^{\mathrm{a}}$ & $71.1^{\mathrm{a}}$ & $69.4^{\mathrm{a}}$ & $73.8^{\mathrm{a}}$ & 73.8 & $69.0^{\mathrm{a}}$ & $70.3^{\mathrm{a}}$ & $73.3^{\mathrm{a}}$ \\
\hline $\mathrm{TiO}_{2}$ & 0.43 & 0.41 & 0.41 & 0.38 & 0.04 & 0.05 & $0.23^{\mathrm{a}}$ & $0.24^{\mathrm{a}}$ & $0.30^{\mathrm{a}}$ & $0.18^{\mathrm{a}}$ & 0.10 & $0.30^{\mathrm{a}}$ & $0.35^{\mathrm{a}}$ & 0.19 \\
\hline $\mathrm{Al}_{2} \mathrm{O}_{3}$ & 15.33 & 15.47 & 15.82 & 15.64 & 12.45 & 11.76 & $14.61^{\mathrm{a}}$ & $14.14^{\mathrm{a}}$ & $14.76^{\mathrm{a}}$ & $13.34^{\mathrm{a}}$ & 12.58 & $14.75^{\mathrm{a}}$ & $14.23^{\mathrm{a}}$ & 13.68 \\
\hline $\mathrm{FeO}^{*}$ & 3.07 & 2.90 & 2.92 & 2.75 & 0.42 & 0.43 & $1.92^{\mathrm{a}}$ & $1.80^{\mathrm{a}}$ & $2.31^{\mathrm{a}}$ & $1.67^{\mathrm{a}}$ & 1.19 & $2.52^{\mathrm{a}}$ & $2.61^{\mathrm{a}}$ & 1.52 \\
\hline $\mathrm{MnO}$ & 0.07 & 0.06 & 0.08 & 0.07 & 0.02 & 0.01 & $0.07^{\mathrm{a}}$ & $0.05^{\mathrm{a}}$ & $0.07^{\mathrm{a}}$ & $0.04^{\mathrm{a}}$ & 0.04 & $0.08^{\mathrm{a}}$ & $0.08^{\mathrm{a}}$ & 0.04 \\
\hline $\mathrm{MgO}$ & 1.19 & 1.14 & 1.12 & 1.04 & 0.02 & 0.09 & $0.75^{\mathrm{a}}$ & $0.93^{\mathrm{a}}$ & $1.14^{\mathrm{a}}$ & $0.30^{\mathrm{a}}$ & 0.15 & $0.86^{\mathrm{a}}$ & $2.41^{\mathrm{a}}$ & 0.70 \\
\hline $\mathrm{CaO}$ & 3.10 & 1.64 & 3.12 & 3.13 & 0.45 & 0.05 & $1.82^{\mathrm{a}}$ & $1.17^{\mathrm{a}}$ & $1.48^{\mathrm{a}}$ & $1.09^{\mathrm{a}}$ & 0.86 & $2.39^{\mathrm{a}}$ & $1.11^{\mathrm{a}}$ & 1.43 \\
\hline $\mathrm{Na}_{2} \mathrm{O}$ & 3.02 & 2.87 & 3.19 & 3.21 & 3.55 & 2.67 & $3.48^{\mathrm{a}}$ & $3.07^{\mathrm{a}}$ & $3.34^{\mathrm{a}}$ & $3.26^{\mathrm{a}}$ & 3.36 & $3.34^{\mathrm{a}}$ & $3.27^{\mathrm{a}}$ & 3.09 \\
\hline $\mathrm{K}_{2} \mathrm{O}$ & 3.51 & 4.66 & 3.87 & 3.96 & 5.33 & 5.86 & $3.99^{\mathrm{a}}$ & $4.93^{\mathrm{a}}$ & $4.17^{\mathrm{a}}$ & $4.76^{\mathrm{a}}$ & 4.73 & $3.79^{a}$ & $3.64^{\mathrm{a}}$ & 4.18 \\
\hline $\mathrm{P}_{2} \mathrm{O}_{5}$ & 0.15 & 0.14 & 0.14 & 0.13 & 0.01 & 0.01 & $0.09^{\mathrm{a}}$ & $0.08^{\mathrm{a}}$ & $0.11^{\mathrm{a}}$ & $0.04^{\mathrm{a}}$ & 0.02 & $0.12^{\mathrm{a}}$ & $0.11^{\mathrm{a}}$ & 0.06 \\
\hline LOI & 1.96 & 2.49 & 0.86 & 0.98 & 0.21 & 0.22 & $0.67^{\mathrm{a}}$ & $1.00^{\mathrm{a}}$ & $0.97^{\mathrm{a}}$ & $0.44^{\mathrm{a}}$ & 0.34 & $1.26^{\mathrm{a}}$ & $0.48^{\mathrm{a}}$ & 0.86 \\
\hline Total & 100.3 & 99.6 & 99.7 & 99.6 & 98.6 & 95.8 & $99.2^{\mathrm{a}}$ & $98.5^{\mathrm{a}}$ & $98.0^{\mathrm{a}}$ & $98.9^{\mathrm{a}}$ & 97.2 & $98.4^{\mathrm{a}}$ & $98.6^{\mathrm{a}}$ & $99.1^{\mathrm{a}}$ \\
\hline$(\mathrm{ppm})$ & & & & & & & & & & & & & & \\
\hline V & 51.9 & 54.8 & 51.1 & 51.3 & 14.6 & 15.8 & $28.7^{\mathrm{a}}$ & $27.2^{\mathrm{a}}$ & $30.8^{\mathrm{a}}$ & $18.8^{\mathrm{a}}$ & 14.4 & $32.0^{\mathrm{a}}$ & $37.4^{\mathrm{a}}$ & $29.6^{\mathrm{a}}$ \\
\hline $\mathrm{Cr}$ & 10.3 & 10.9 & 13.1 & 9.5 & 3.3 & 3.3 & $4.2^{\mathrm{a}}$ & $8.1^{\mathrm{a}}$ & $6.0^{\mathrm{a}}$ & $1.4^{\mathrm{a}}$ & 4.8 & $4.5^{\mathrm{a}}$ & $8.7^{\mathrm{a}}$ & $4.5^{\mathrm{a}}$ \\
\hline Co & 9.2 & 10.8 & 9.0 & 7.9 & 3.1 & 2.5 & $6.1^{\mathrm{a}}$ & $5.9^{\mathrm{a}}$ & $7.3^{\mathrm{a}}$ & $6.8^{\mathrm{a}}$ & 5.5 & $7.1^{\mathrm{a}}$ & $9.2^{\mathrm{a}}$ & $4.7^{\mathrm{a}}$ \\
\hline $\mathrm{Ni}$ & 4.4 & 4.7 & 4.8 & 3.6 & 4.5 & 4.6 & $4.4^{\mathrm{a}}$ & $5.7^{\mathrm{a}}$ & $5.3^{\mathrm{a}}$ & $3.6^{\mathrm{a}}$ & 6.2 & $3.3^{\mathrm{a}}$ & $4.9^{\mathrm{a}}$ & $5.1^{\mathrm{a}}$ \\
\hline $\mathrm{Cu}$ & 1.2 & n.d. & 2.2 & n.d. & n.d. & 0.2 & $1.4^{\mathrm{a}}$ & n.d. ${ }^{\mathrm{a}}$ & n.d. ${ }^{\mathrm{a}}$ & n.d. ${ }^{a}$ & n.d. & $1.6^{\mathrm{a}}$ & n.d. ${ }^{\mathrm{a}}$ & $5.0^{\mathrm{a}}$ \\
\hline $\mathrm{Zn}$ & 51.8 & 44.3 & 47.8 & 51.4 & 14.9 & 9.3 & $42.9^{\mathrm{a}}$ & $48.6^{\mathrm{a}}$ & $49.3^{\mathrm{a}}$ & $24.8^{\mathrm{a}}$ & 20.3 & $49.0^{\mathrm{a}}$ & $49.8^{\mathrm{a}}$ & $27.2^{\mathrm{a}}$ \\
\hline $\mathrm{Rb}$ & 101 & 163 & 121 & 116 & 263 & 189 & $154^{\mathrm{a}}$ & $172^{\mathrm{a}}$ & $147^{\mathrm{a}}$ & $284^{\mathrm{a}}$ & 305 & $114^{\mathrm{a}}$ & $152^{\mathrm{a}}$ & $153^{\mathrm{a}}$ \\
\hline $\mathrm{Sr}$ & 383 & 322 & 401 & 380 & 9.7 & 36.6 & $266^{\mathrm{a}}$ & $184^{\mathrm{a}}$ & $259^{\mathrm{a}}$ & $95.8^{\mathrm{a}}$ & 60.4 & $317^{\mathrm{a}}$ & $247^{\mathrm{a}}$ & $231^{\mathrm{a}}$ \\
\hline Y & 18.9 & 19.9 & 20.2 & 18.6 & 32.3 & 15.3 & $25.1^{\mathrm{a}}$ & $21.8^{\mathrm{a}}$ & $21.7^{\mathrm{a}}$ & $43.8^{\mathrm{a}}$ & 49.3 & $21.1^{\mathrm{a}}$ & $26.3^{\mathrm{a}}$ & $21.3^{\mathrm{a}}$ \\
\hline $\mathrm{Zr}$ & 171 & 162 & 153 & 149 & 83.6 & 27.6 & $126^{\mathrm{a}}$ & $153^{a}$ & $147^{\mathrm{a}}$ & $164^{\mathrm{a}}$ & 103 & $141^{\mathrm{a}}$ & $147^{\mathrm{a}}$ & $91.5^{\mathrm{a}}$ \\
\hline $\mathrm{Nb}$ & 4.5 & 4.0 & 4.1 & 3.3 & 9.0 & n.d. & $5.2^{\mathrm{a}}$ & $5.7^{\mathrm{a}}$ & $4.8^{\mathrm{a}}$ & $7.3^{\mathrm{a}}$ & 4.4 & $5.3^{\mathrm{a}}$ & $6.6^{\mathrm{a}}$ & $2.7^{\mathrm{a}}$ \\
\hline $\mathrm{Ba}$ & 567 & 711 & 640 & 669 & 9.0 & n.d. & $481^{\mathrm{a}}$ & $630^{\mathrm{a}}$ & $586^{\mathrm{a}}$ & $574^{\mathrm{a}}$ & 303 & $548^{\mathrm{a}}$ & $381^{\mathrm{a}}$ & $387^{\mathrm{a}}$ \\
\hline $\mathrm{Pb}$ & 15.0 & 19.8 & 17.3 & 17.7 & 49.5 & 32.0 & $24.7^{\mathrm{a}}$ & $27.9^{\mathrm{a}}$ & $21.4^{\mathrm{a}}$ & $18.9^{\mathrm{a}}$ & 22.9 & $20.8^{\mathrm{a}}$ & $17.0^{\mathrm{a}}$ & $18.8^{\mathrm{a}}$ \\
\hline Th & 12.7 & 10.3 & 9.7 & 10.1 & 34.4 & 9.8 & $18.3^{\mathrm{a}}$ & $18.2^{\mathrm{a}}$ & $12.6^{\mathrm{a}}$ & $26.3^{\mathrm{a}}$ & 23.8 & $12.0^{\mathrm{a}}$ & $19.9^{\mathrm{a}}$ & $19.2^{\mathrm{a}}$ \\
\hline $\mathrm{Y}$ & 13.0 & & 11.7 & 10.7 & & & 16.7 & 12.9 & 13.6 & 34.8 & & 137 & 17.4 & 12.8 \\
\hline $\mathrm{La}$ & 35.3 & & 17.3 & 20.7 & & & 21.9 & 31.8 & 24.3 & 23.9 & & 21.0 & 23.2 & 30.7 \\
\hline $\mathrm{Ce}$ & 65.3 & & 31.1 & 39.7 & & & 42.7 & 61.5 & 47.6 & 67.2 & & 41.5 & 46.0 & 58.7 \\
\hline $\operatorname{Pr}$ & 6.55 & & 3.44 & 4.16 & & & 4.57 & 6.37 & 5.09 & 7.28 & & 4.52 & 4.96 & 6.04 \\
\hline $\mathrm{Nd}$ & 22.5 & & 12.5 & 14.5 & & & 16.2 & 22.0 & 17.8 & 25.0 & & 16.1 & 17.8 & 20.1 \\
\hline $\mathrm{Sm}$ & 3.76 & & 2.34 & 2.63 & & & 3.20 & 3.83 & 3.23 & 5.06 & & 3.04 & 3.56 & 3.34 \\
\hline $\mathrm{Eu}$ & 0.99 & & 0.77 & 0.77 & & & 0.68 & 0.78 & 0.75 & 0.64 & & 0.79 & 0.70 & 0.69 \\
\hline $\mathrm{Gd}$ & 3.07 & & 2.04 & 2.17 & & & 2.76 & 3.22 & 2.72 & 4.63 & & 2.58 & 2.96 & 2.70 \\
\hline $\mathrm{Tb}$ & 0.42 & & 0.30 & 0.31 & & & 0.45 & 0.45 & 0.40 & 0.80 & & 0.40 & 0.46 & 0.36 \\
\hline Dy & 2.32 & & 1.78 & 1.78 & & & 2.66 & 2.35 & 2.27 & 5.20 & & 2.30 & 2.82 & 2.03 \\
\hline Ho & 0.45 & & 0.36 & 0.34 & & & 0.54 & 0.43 & 0.44 & 1.11 & & 0.46 & 0.57 & 0.41 \\
\hline $\mathrm{Er}$ & 1.31 & & 1.08 & 1.02 & & & 1.60 & 1.21 & 1.29 & 3.32 & & 1.36 & 1.70 & 1.24 \\
\hline $\mathrm{Tm}$ & 0.19 & & 0.15 & 0.15 & & & 0.24 & 0.18 & 0.19 & 0.50 & & 0.19 & 0.25 & 0.19 \\
\hline $\mathrm{Yb}$ & 1.25 & & 1.02 & 0.97 & & & 1.63 & 1.09 & 1.26 & 3.24 & & 1.34 & 1.65 & 1.23 \\
\hline $\mathrm{Lu}$ & 0.19 & & 0.16 & 0.15 & & & 0.25 & 0.15 & 0.18 & 0.44 & & 0.19 & 0.25 & 0.18 \\
\hline $\mathrm{La}_{\mathrm{N}} / \mathrm{Sm}_{\mathrm{N}}$ & 5.87 & & 4.62 & 4.92 & & & 4.28 & 5.20 & 4.72 & 4.08 & & 4.32 & 4.08 & 5.76 \\
\hline $\mathrm{Gd}_{\mathrm{N}} / \mathrm{Yb}_{\mathrm{N}}$ & 2.03 & & 1.66 & 1.85 & & & 1.40 & 2.44 & 1.78 & 1.18 & & 1.59 & 1.48 & 1.82 \\
\hline $\mathrm{La}_{\mathrm{N}} / \mathrm{Yb}_{\mathrm{N}}$ & 19.5 & & 11.8 & 14.8 & & & 9.32 & 20.2 & 13.4 & 7.04 & & 10.8 & 9.70 & 17.3 \\
\hline $\mathrm{Eu} / \mathrm{Eu}^{\#}$ & 0.88 & & 1.07 & 0.98 & & & 0.70 & 0.67 & 0.77 & 0.40 & & 0.85 & 0.66 & 0.70 \\
\hline$\sum$ REE & 144 & & 74 & 89 & & & 99 & 135 & 108 & 157 & & 96 & 107 & 128 \\
\hline
\end{tabular}

$\mathrm{FeO} *$, total iron represented as FeO. n.d., not detected. N, normalized value by CI Chondrites of Anders and Grevesse (1989) and Shinotsuka et al. (1995). $E u^{\#}=(S m-G d)^{\wedge}(1 / 2)$.

${ }^{a}$ Data from Tanioka et al. (2014).

The REE concentrations of the Tadamigawa olderstage granites are provided in Table 2, and the REE patterns normalized by $\mathrm{C} 1$ chondrite data (Anders and Grevesse, 1989; Shinotsuka et al., 1995) are shown in Fig. 4. Data from Tanioka et al. (2014) are also included in Fig. 4. These granites had a large variation in both the total REE content $\left(\sum \mathrm{REE}=74-163 \mathrm{ppm}\right)$ and the degree of the Eu anomaly $\left(\mathrm{Eu} / \mathrm{Eu}^{\#} ; \mathrm{Eu}^{\#}=\left(\mathrm{Sm}_{\mathrm{N}} \times \mathrm{Gd}_{\mathrm{N}}\right)^{\wedge}(1 / 2)\right.$, 0.26-1.07). All of the granites had LREE-enriched patterns $\left(\mathrm{La}_{\mathrm{N}} / \mathrm{Yb}_{\mathrm{N}}=2.76-20.8\right)$. The Eu anomalies of the Hinoematagawa granites $\left(\mathrm{Eu} / \mathrm{Eu}^{\#}=0.73-1.01\right)$ were small (Figs. 4A and B). On the other hand, the degrees of $\mathrm{Eu}$ anomaly for the Tadamigawa granites varied from 0.26 to 0.98 and are larger than those for the Hinoematagawa 


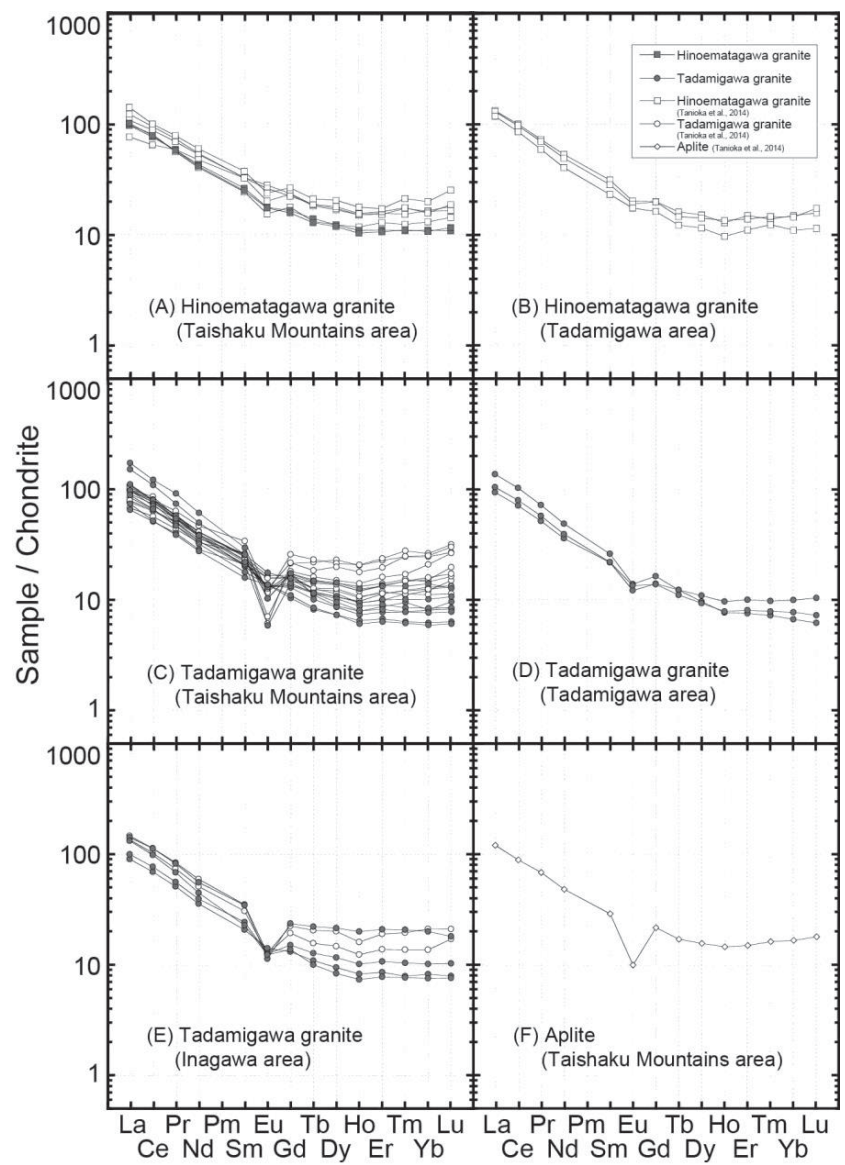

Fig. 4. Rare earth elements patterns of the Tadamigawa olderstage granites, normalized by the C1 chondrite data of Anders and Grevesse (1989) and Shinotsuka et al. (1995). (A) The Hinoematagawa granite from the Taishaku Mountains area, (B) the Hinoematagawa granite from the Tadamigawa area, $(C)$ the Tadamigawa granite from the Taishaku Mountains area, (D) the Tadamigawa granite from the Tadamigawa area, $(E)$ the Tadamigawa granite from the Inagawa area, and $(F)$ aplite in the Tadamigawa granite from the Taishaku Mountains area. A part of the data from Tanioka et al. (2014).

granites. These results were consistent with the results from Tanioka et al. (2014). Moreover, the Tadamigawa granites in the Tadamigawa area have similar REE patterns as those of the Hinoematagawa granites.

\section{$R b$-Sr whole-rock isochron and mineral isochron}

The ${ }^{87} \mathrm{Sr} /{ }^{86} \mathrm{Sr}$ and ${ }^{87} \mathrm{Rb} /{ }^{86} \mathrm{Sr}$ ratios of the Tadamigawa older-stage granites are shown in Table 3 . In addition, the biotite-included and the biotite-excluded isochron ages of the Tadamigawa older-stage granites in the Taishaku Mountains area are shown in Table 4 , and the ${ }^{87} \mathrm{Rb} /{ }^{86} \mathrm{Sr}$ versus ${ }^{87} \mathrm{Sr} /{ }^{86} \mathrm{Sr}$ diagrams for whole-rocks and minerals in the Taishaku Mountains and the Inagawa area are provided in Figs. 5, 6 and 7. Isochrons were calculated using
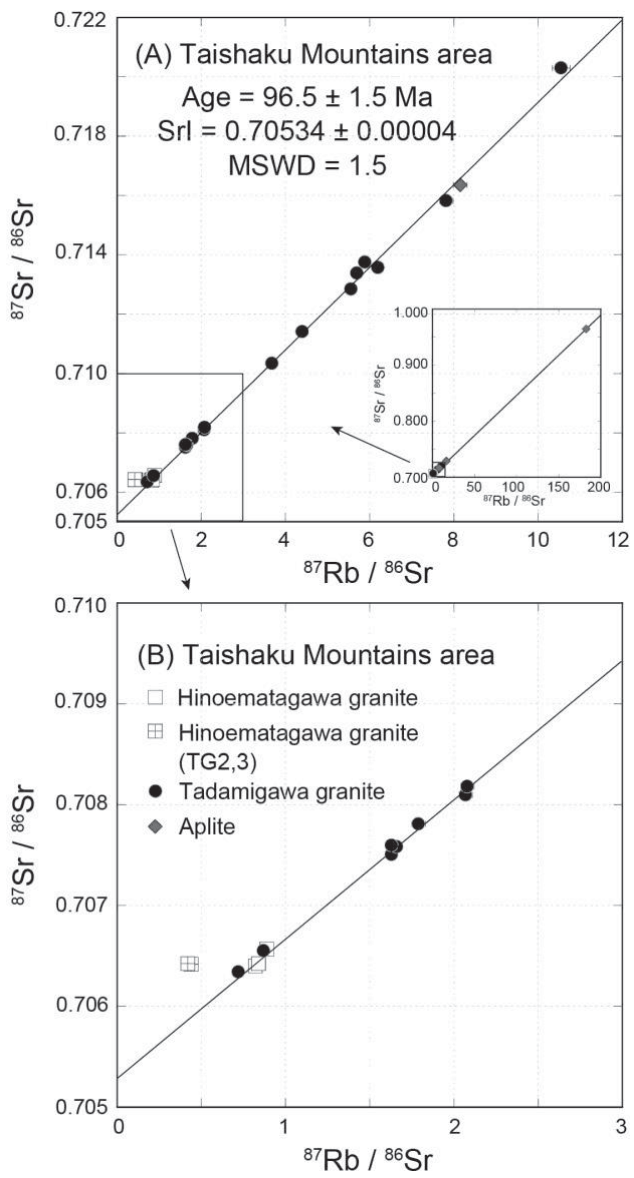

Fig. 5. (A) ${ }^{87} \mathrm{Rb} /{ }^{86} \mathrm{Sr}$ versus ${ }^{87} \mathrm{Sr} /{ }^{86} \mathrm{Sr}$ diagrams of the Tadamigawa older-stage granites whole-rock samples from the Taishaku Mountains area. A whole-rock isochron was calculated from 16 samples for the Tadamigawa granite. (B) Extended figure of $A$.

the regression program of ISOPLOT version 3.70 (Ludwig, 2008), considering 4\% errors in ${ }^{87} \mathrm{Rb} /{ }^{86} \mathrm{Sr}$ and each analytical error in ${ }^{87} \mathrm{Sr} /{ }^{86} \mathrm{Sr}$ (Table 3).

The whole-rock samples of the Tadamigawa granite in the Taishaku Mountains area showed a well-correlated single trend, indicating that the $\mathrm{Rb}-\mathrm{Sr}$ system of the Tadamigawa granite in the Taishaku Mountains area is isochronous on a pluton scale. The Tadamigawa granite whole-rock isochron yielded an age of $96.5 \pm 1.5 \mathrm{Ma}(2 \sigma)$ and $\mathrm{SrI}=0.70534 \pm 0.00004(\mathrm{MSWD}=1.5, \mathrm{n}=16)$ (Fig. 5A). The Hinoematagawa granites, except for two samples (TG2, 3), are plotted on this Rb-Sr whole-rock isochron (Fig. 5B). The two exception samples had different whole-rock chemical compositions than the other samples, especially in trace elements: they had lower $\mathrm{V}$ and $\mathrm{Cr}$ and higher $\mathrm{Sr}$ and $\mathrm{Ca}$ contents than the other samples (Fig. 3).

The $\mathrm{Rb}-\mathrm{Sr}$ mineral isochron ages for six samples 
Table 3. Analytical data for Rb and Sr of the Tadamigawa older-stage granites

\begin{tabular}{|c|c|c|c|c|c|c|c|c|c|}
\hline Area & Rock type & Sample & $\begin{array}{c}\mathrm{Rb} \\
(\mathrm{ppm})^{*}\end{array}$ & $\begin{array}{c}\mathrm{Sr} \\
(\mathrm{ppm})^{*}\end{array}$ & ${ }^{87} \mathrm{Rb} /{ }^{86} \mathrm{Sr}$ & ${ }^{87} \mathrm{Sr} /{ }^{86} \mathrm{Sr}$ & $(2 \sigma)$ & $\begin{array}{c}\varepsilon_{\mathrm{Sr}} \\
(100 \mathrm{Ma})\end{array}$ & $\begin{array}{c}\varepsilon_{\mathrm{Sr}} \\
(60 \mathrm{Ma})\end{array}$ \\
\hline \multirow[t]{43}{*}{ Taishaku Mountains area } & \multirow[t]{9}{*}{ Hinoematagawa granite } & TG1a & 127 & 410 & 0.89 & 0.706567 & $(2)$ & \multirow[t]{5}{*}{+13.0} & \\
\hline & & $-\mathrm{Pl}$ & 25.6 & 890 & 0.08 & 0.705410 & (2) & & \\
\hline & & -Kfs & 260 & 399 & 1.89 & 0.707828 & (3) & & \\
\hline & & $-\mathrm{Bt} 1$ & 450 & 18.1 & 71.9 & 0.805330 & $(2)$ & & \\
\hline & & $-\mathrm{Bt} 2$ & 460 & 18.3 & 72.7 & 0.805608 & (4) & & \\
\hline & & TG2 & 92.5 & 605 & 0.44 & 0.706417 & (2) & +20.0 & \\
\hline & & TG3 & 90.2 & 626 & 0.42 & 0.706425 & (3) & +20.4 & \\
\hline & & TG10 & 110 & 389 & 0.82 & 0.706402 & $(2)$ & +12.2 & \\
\hline & & TG36 & 123 & 420 & 0.84 & 0.706425 & $(2)$ & +12.1 & \\
\hline & \multirow[t]{31}{*}{ Tadamigawa granite } & TG4b & 138 & 221 & 1.79 & 0.707809 & $(2)$ & +12.4 & \\
\hline & & TG5 & 171 & 239 & 2.07 & 0.708096 & $(2)$ & +10.9 & \\
\hline & & $-\mathrm{Pl}$ & 13.9 & 557 & 0.07 & 0.705422 & (2) & & \\
\hline & & $-\mathrm{Kfs}$ & 255 & 514 & 1.44 & 0.707235 & (2) & & \\
\hline & & $-B t$ & 657 & 13.8 & 138 & 0.895373 & (3) & & \\
\hline & & TG6 & 135 & 187 & 2.08 & 0.708180 & (2) & +12.0 & \\
\hline & & TG7 & 154 & 278 & 1.63 & 0.707504 & (2) & +11.4 & \\
\hline & & TG8 & 114 & 198 & 1.66 & 0.707583 & (2) & +12.0 & \\
\hline & & TG9b & 232 & 121 & 5.56 & 0.712845 & (2) & +8.0 & \\
\hline & & TG9e & 155 & 78.4 & 5.70 & 0.713384 & $(5)$ & +12.7 & \\
\hline & & $-\mathrm{Pl}$ & 22 & 142 & 0.45 & 0.705960 & (2) & & \\
\hline & & -Kfs & 314 & 103 & 8.86 & 0.717562 & (2) & & \\
\hline & & $-B t$ & 688 & 12.9 & 154 & 0.895297 & $(2)$ & & \\
\hline & & TG11 & 158 & 77.1 & 5.89 & 0.713753 & (2) & +14.3 & \\
\hline & & TG12 & 244 & 89.7 & 7.82 & 0.715821 & (2) & +4.6 & \\
\hline & & $-\mathrm{Kfs} 1$ & 366 & 131 & 8.09 & 0.716374 & (3) & & \\
\hline & & $-\mathrm{Kfs} 2$ & 348 & 474 & 2.13 & 0.708180 & (2) & & \\
\hline & & $-B t$ & 550 & 55.5 & 28.7 & 0.742753 & (2) & & \\
\hline & & TG13 & 268 & 174 & 4.40 & 0.711409 & $(2)$ & +11.0 & \\
\hline & & -Pl1 & 87.3 & 435 & 0.58 & 0.706220 & (2) & & \\
\hline & & $-\mathrm{Pl} 2$ & 280 & 267 & 3.03 & 0.709422 & (2) & & \\
\hline & & $-\mathrm{Kfs}$ & 401 & 432 & 2.68 & 0.708943 & (2) & & \\
\hline & & $-B t$ & 1020 & 25.3 & 117 & 0.844316 & (2) & & \\
\hline & & TG40 & 164 & 44.9 & 10.5 & 0.720297 & (3) & +13.1 & \\
\hline & & $-\mathrm{Pl}$ & 41.2 & 60.7 & 1.96 & 0.708081 & (2) & & \\
\hline & & $-\mathrm{Kfs}$ & 416 & 48.9 & 24.7 & 0.738619 & (2) & & \\
\hline & & TG41 & 128 & 100 & 3.68 & 0.710340 & (2) & +10.2 & \\
\hline & & TG42 & 175 & 81.8 & 6.20 & 0.713567 & $(2)$ & +5.2 & \\
\hline & & TG43 & 151 & 266 & 1.63 & 0.707596 & $(2)$ & +12.8 & \\
\hline & & TG46a & 94.4 & 377 & 0.72 & 0.706342 & $(2)$ & +13.2 & \\
\hline & & TG48 & 119 & 392 & 0.87 & 0.706551 & $(2)$ & +13.2 & \\
\hline & \multirow[t]{3}{*}{ Aplite } & TG9a & 147 & 52.3 & 8.15 & 0.716355 & (2) & +5.4 & \\
\hline & & TG9c & 255 & 4.3 & 183 & 0.964546 & (3) & +11.1 & \\
\hline & & TG9d & 194 & 33.7 & 16.6 & 0.728982 & $(2)$ & +13.8 & \\
\hline Inagawa area & Tadamigawa granite & TG29b & 299 & 55.9 & 15.5 & 0.722118 & (4) & & +64.0 \\
\hline
\end{tabular}

*Data analyzed by ICP-MS. Abbreviations are: Pl, plagioclase; Kfs, K-feldspar; Bt, biotite.

(TG1a, 5, 9e, 12, 13 and 40) of the Tadamigawa olderstage granites were interpreted as 92.7 to $98.1 \mathrm{Ma}$, and are similar to or slightly younger than the $\mathrm{Rb}-\mathrm{Sr}$ wholerock isochron age. Biotite showed a high $\mathrm{Rb} / \mathrm{Sr}$ ratio in the $\mathrm{Rb}-\mathrm{Sr}$ system, and it is not resistant to $\mathrm{Sr}$ isotopic disturbance by low-temperature processes such as retrogressive metamorphism or secondary weathering. Altered or weathered biotite may disturb the $\mathrm{Rb}-\mathrm{Sr}$ mineral isochron, yielding younger mineral isochron ages. There- fore, two types of the Rb-Sr mineral isochron were calculated: biotite-included isochron and biotite-excluded isochron (Table 4). The Rb-Sr mineral isochrons of two samples (TG1a, 12) had no difference between the biotiteincluded isochron and the biotite-excluded isochron. On the other hand, the biotite-excluded isochrons of three samples (TG5, 9e, 13) showed better MSWD values than the biotite-included isochrons (Table 4). 
Table 4. Biotite-included and-excluded Rb-Sr mineral isochron ages of the Tadamigawa older-stage granites in the Taishaku Mountains area

\begin{tabular}{llcccccc}
\hline Rock type & Sample & Age (Ma) & $\begin{array}{c}\text { Bt-included } \\
\text { SrI }(2 \sigma)\end{array}$ & MSWD & Age (Ma) & $\begin{array}{c}\text { Bt-excluded } \\
\text { SrI }(2 \sigma)\end{array}$ & MSWD \\
\hline Hinoematagawa granite & TG1a & $97.3 \pm 2.0$ & $0.70530(1)$ & 1.3 & $95 \pm 41$ & $0.70532(59)$ & 3.9 \\
& & & & & & & \\
Tadamigawa granite & TG12 & $93.5 \pm 2.8$ & $0.70537(16)$ & 1.2 & $95.2 \pm 3.8$ & $0.70530(19)$ & 0.7 \\
& TG40* & & & & $97.1 \pm 3.1$ & $0.70538(15)$ & 3.0 \\
& TG5 & $95.0 \pm 2.2$ & $0.70532(1)$ & 1.0 & $93.9 \pm 2.7$ & $0.70533(1)$ & 0.1 \\
& TG9e & $86.9 \pm 7.7$ & $0.7061(18)$ & 12 & $98.1 \pm 2.9$ & $0.70534(3)$ & 0.5 \\
& TG13 & $84.4 \pm 5.3$ & $0.70576(43)$ & 5.8 & $92.7 \pm 2.7$ & $0.70545(4)$ & 0.6 \\
\hline
\end{tabular}

*Biotite fraction was not measured. Abbreviations is: Bt, biotite.

\section{Zircon $U$-Pb age}

The separated zircon grains contained no inclusion and showed oscillatory zoning under CL. The results of the LA-ICP-MS analyses are shown in Table 5 and Fig. 8. High Th/U ratios (0.22-1.01) confirm the magmatic origin for the zircon minerals.

The ${ }^{207} \mathrm{~Pb} /{ }^{235} \mathrm{U}-{ }^{206} \mathrm{~Pb} /{ }^{238} \mathrm{U}$ concordia diagrams give $\mathrm{U}-\mathrm{Pb}$ ages of $101.1 \pm 2.1 \mathrm{Ma}(95 \%$ conf., $\mathrm{MSWD}=1.7)$ for TG36, $106.7 \pm 0.6 \mathrm{Ma}(95 \%$ conf., MSWD $=2.0)$ for TG43, $103.1 \pm 1.8 \mathrm{Ma}(95 \%$ conf., MSWD $=2.8$ ) for TG20 and $62.2 \pm 0.7 \mathrm{Ma}(95 \%$ conf., MSWD $=2.5)$ for TG29b. The U-Pb ages of TG36 (Hinoematagawa granite) and TG43 (Tadamigawa granite) samples in the Taishaku Mountains area are similar to each other and are also similar to the age of TG20 (Tadamigawa granite) in the Tadamigawa area. The age of TG29b (Tadamigawa granite) in the Inagawa area is younger than those of the samples in the other areas.

\section{DISCUSSION}

Crystallization differentiation of the Tadamigawa olderstage granites

Most of the elements showed a single chemical trend on the Harker diagram (Fig. 3). These results indicate that the Himoematagawa and the Tadamigawa granites were formed by the differentiation of the same single parental magma and that the Tadamigawa granite is more evolved than the Hinoematagawa granite (Tanioka et al., 2014). There was no significant difference in whole-rock chemical composition between the two plutons except for rock type. The degree of Eu anomaly increased with the degree of crystallization differentiation. The negative $\mathrm{Eu}$ anomaly might have occurred by the removal of plagioclase, which concentrates Eu (Bea, 1996). In addition, the ${ }^{87} \mathrm{Rb} /{ }^{86} \mathrm{Sr}$ ratios of the Tadamigawa granite were higher than those of the Hinoematagawa granite (Fig. 5A). All of the evidence shows that the Tadamigawa granite might be differentiated from the Hinoematagawa granite.
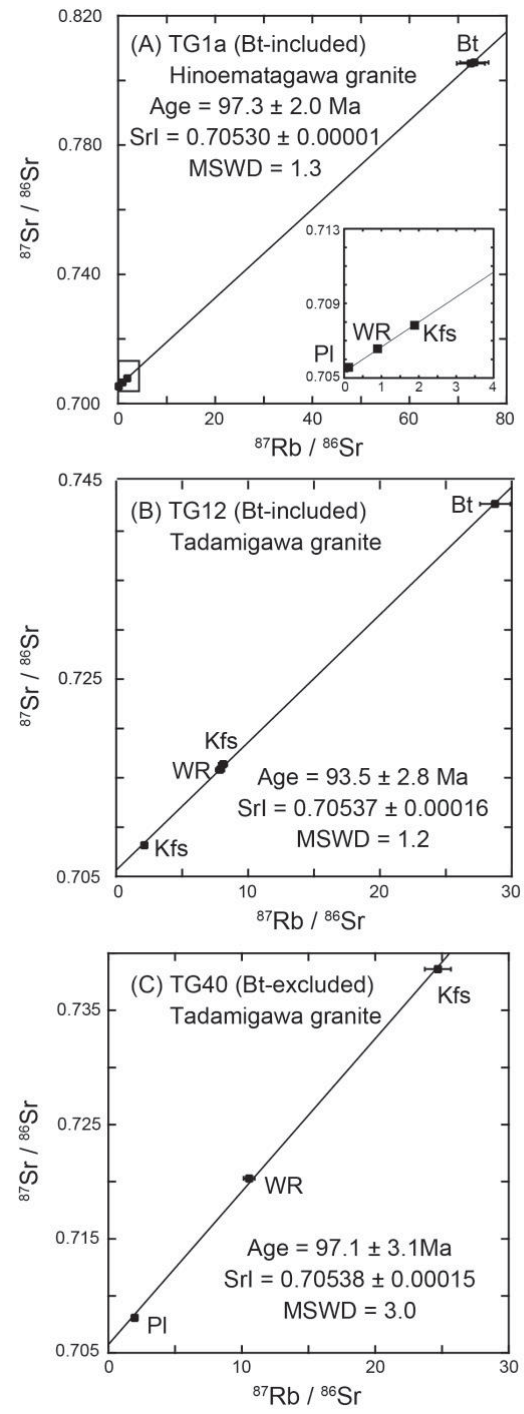

Fig. 6. ${ }^{87} \mathrm{Rb} /{ }^{86} \mathrm{Sr}$ versus ${ }^{87} \mathrm{Sr} /{ }^{86} \mathrm{Sr}$ diagrams of the granites of the Taishaku Mountains area: (A) a Hinoematagawa granite TG1a, (B) a Tadamigawa granite TG12 and (C) a Tadamigawa granite TG40. Isochron ages were calculated with biotite samples. Abbreviations are: $\mathrm{Pl}$, plagioclase; $\mathrm{Kfs}$, $\mathrm{K}$-feldspar; $\mathrm{Bt}$, biotite; WR, whole-rock. 



Fig. 7. (A-C) ${ }^{87} \mathrm{Rb} /{ }^{86} \mathrm{Sr}$ versus ${ }^{87} \mathrm{Sr} /{ }^{86} \mathrm{Sr}$ diagrams of Tadamigawa granites, TG5, TG9e and TG13 of the Tadamigawa granite from the Taishaku Mountains area. Isochron ages were calculated excluding the biotite samples. $(D-F)$ Deviation of the mineral data points from the biotite-excluded isochron plotted against ${ }^{87} \mathrm{Rb} /{ }^{86} \mathrm{Sr}$ ratio. The deviation was calculated as $/\left({ }^{87} \mathrm{Sr} /{ }^{86} \mathrm{Sr}\right)_{a c t}-$ $\left({ }^{87} \mathrm{Sr} /{ }^{86} \mathrm{Sr}\right)_{\text {iso }} / 1 / 10^{4}$, where $\left({ }^{87} \mathrm{Sr} /{ }^{86} \mathrm{Sr}\right)_{\text {act }}$ is the measured ${ }^{87} \mathrm{Sr} /{ }^{86} \mathrm{Sr}$ ratio of the mineral and $\left({ }^{87} \mathrm{Sr} /{ }^{86} \mathrm{Sr}\right)_{\text {iso }}$ is the ${ }^{87} \mathrm{Sr} /{ }^{86} \mathrm{Sr}$ ratio of isochron for a given ${ }^{87} \mathrm{Rb} /{ }^{86} \mathrm{Sr}$ ratio of mineral. Abbreviations are: Pl, plagioclase; $\mathrm{Kfs}$, $\mathrm{K}$-feldspar; WR, whole-rock.

$R b-S r$ whole-rock ages and mineral isochron ages

The Tadamigawa granite whole-rock samples in the Taishaku Mountains area form a well constrained isochron in the $\mathrm{Rb}$-Sr isochron plot (Fig. 5A). The aplite and the Hinoematagawa granite, except for two samples (TG2, 3 ), were plotted perfectly on this whole-rock isochron (Fig. 5B). This indicates that the aplite samples and the
Hinoematagawa granites were simultaneously formed from the same magma source as the Tadamigawa granites. Two samples of the Hinoematagawa granites in the Taishaku Mountains area (TG2, 3) plotted away the Tadamigawa granite whole-rock isochron, showing ${ }^{87} \mathrm{Sr} /$ ${ }^{86} \mathrm{Sr}$ ratios higher than the isochron line (Fig. 5B). These two samples had different chemical composition trends 
Table 5. U-Pb isotope data for zircon crystal determined by LA-ICP-MS

\begin{tabular}{|c|c|c|c|c|c|c|c|}
\hline \multirow[t]{2}{*}{ Sample } & \multirow[t]{2}{*}{ Spot No. } & \multirow[t]{2}{*}{$\mathrm{Th} / \mathrm{U}$} & \multicolumn{3}{|c|}{ Isotopic ratios } & \multicolumn{2}{|c|}{ Age (Ma) } \\
\hline & & & ${ }^{207} \mathrm{~Pb} /{ }^{206} \mathrm{~Pb}(2 \sigma)$ & ${ }^{206} \mathrm{~Pb} /{ }^{238} \mathrm{U}(2 \sigma)$ & ${ }^{207} \mathrm{~Pb} /{ }^{235} \mathrm{U}(2 \sigma)$ & ${ }^{206} \mathrm{~Pb} /{ }^{238} \mathrm{U}(2 \sigma)$ & ${ }^{207} \mathrm{~Pb} /{ }^{235} \mathrm{U}(2 \sigma)$ \\
\hline \multirow[t]{7}{*}{ TG36 } & 1 & 1.01 & $0.0500 \pm 0.0031$ & $0.0156 \pm 0.0004$ & $0.108 \pm 0.007$ & $100.1 \pm 2.7$ & $104.0 \pm 7.0$ \\
\hline & 2 & 0.49 & $0.0548 \pm 0.0065$ & $0.0157 \pm 0.0006$ & $0.119 \pm 0.015$ & $100.7 \pm 3.6$ & $114.1 \pm 14.2$ \\
\hline & 3 & 0.50 & $0.0473 \pm 0.0060$ & $0.0165 \pm 0.0006$ & $0.108 \pm 0.014$ & $105.7 \pm 3.8$ & $104.0 \pm 13.8$ \\
\hline & 4 & 0.52 & $0.0519 \pm 0.0055$ & $0.0156 \pm 0.0005$ & $0.112 \pm 0.012$ & $99.7 \pm 3.3$ & $107.5 \pm 12.0$ \\
\hline & 5 & 0.52 & $0.0511 \pm 0.0054$ & $0.0160 \pm 0.0004$ & $0.112 \pm 0.012$ & $102.0 \pm 2.8$ & $108.1 \pm 11.8$ \\
\hline & 6 & 0.50 & $0.0491 \pm 0.0059$ & $0.0156 \pm 0.0005$ & $0.106 \pm 0.013$ & $99.9 \pm 2.9$ & $102.0 \pm 12.6$ \\
\hline & & & & & Average & $101.1 \pm 2.1$ & $105.8 \pm 4.3$ \\
\hline \multirow[t]{25}{*}{ TG43 } & 1 & 0.37 & $0.0487 \pm 0.0032$ & $0.0170 \pm 0.0004$ & $0.114 \pm 0.008$ & $108.6 \pm 2.4$ & $109.7 \pm 7.6$ \\
\hline & 2 & 0.31 & $0.0521 \pm 0.0041$ & $0.0169 \pm 0.0004$ & $0.121 \pm 0.010$ & $107.9 \pm 2.6$ & $116.1 \pm 9.5$ \\
\hline & 3 & 0.64 & $0.0506 \pm 0.0031$ & $0.0170 \pm 0.0004$ & $0.118 \pm 0.008$ & $108.4 \pm 2.4$ & $113.4 \pm 7.4$ \\
\hline & 4 & 0.37 & $0.0470 \pm 0.0030$ & $0.0168 \pm 0.0003$ & $0.109 \pm 0.007$ & $107.3 \pm 2.0$ & $104.9 \pm 6.9$ \\
\hline & 5 & 0.44 & $0.0512 \pm 0.0035$ & $0.0167 \pm 0.0003$ & $0.118 \pm 0.008$ & $106.8 \pm 2.1$ & $113.1 \pm 8.1$ \\
\hline & 6 & 0.30 & $0.0503 \pm 0.0037$ & $0.0168 \pm 0.0003$ & $0.117 \pm 0.009$ & $107.4 \pm 2.2$ & $112.0 \pm 8.6$ \\
\hline & 7 & 0.36 & $0.0517 \pm 0.0034$ & $0.0165 \pm 0.0003$ & $0.118 \pm 0.008$ & $105.6 \pm 2.0$ & $113.0 \pm 7.8$ \\
\hline & 8 & 0.37 & $0.0493 \pm 0.0041$ & $0.0164 \pm 0.0003$ & $0.112 \pm 0.009$ & $105.0 \pm 2.0$ & $107.4 \pm 9.1$ \\
\hline & 9 & 0.47 & $0.0492 \pm 0.0035$ & $0.0164 \pm 0.0003$ & $0.111 \pm 0.008$ & $104.9 \pm 1.8$ & $107.2 \pm 7.9$ \\
\hline & 10 & 0.56 & $0.0493 \pm 0.0048$ & $0.0164 \pm 0.0004$ & $0.112 \pm 0.011$ & $105.1 \pm 2.3$ & $107.4 \pm 10.7$ \\
\hline & 11 & 0.77 & $0.0485 \pm 0.0033$ & $0.0166 \pm 0.0003$ & $0.111 \pm 0.008$ & $105.8 \pm 2.2$ & $106.6 \pm 7.6$ \\
\hline & 12 & 0.43 & $0.0503 \pm 0.0033$ & $0.0170 \pm 0.0003$ & $0.118 \pm 0.008$ & $108.9 \pm 2.2$ & $113.4 \pm 7.7$ \\
\hline & 13 & 0.36 & $0.0469 \pm 0.0044$ & $0.0168 \pm 0.0004$ & $0.109 \pm 0.010$ & $107.3 \pm 2.6$ & $104.6 \pm 10.1$ \\
\hline & 14 & 0.38 & $0.0455 \pm 0.0044$ & $0.0168 \pm 0.0004$ & $0.105 \pm 0.010$ & $107.2 \pm 2.6$ & $101.6 \pm 10.1$ \\
\hline & 15 & 0.34 & $0.0454 \pm 0.0037$ & $0.0169 \pm 0.0004$ & $0.106 \pm 0.009$ & $108.0 \pm 2.4$ & $102.1 \pm 8.7$ \\
\hline & 16 & 0.36 & $0.0523 \pm 0.0050$ & $0.0168 \pm 0.0004$ & $0.121 \pm 0.012$ & $107.6 \pm 2.6$ & $116.4 \pm 11.4$ \\
\hline & 17 & 0.36 & $0.0504 \pm 0.0046$ & $0.0164 \pm 0.0004$ & $0.114 \pm 0.011$ & $104.7 \pm 2.4$ & $109.4 \pm 10.3$ \\
\hline & 18 & 0.59 & $0.0499 \pm 0.0026$ & $0.0164 \pm 0.0003$ & $0.113 \pm 0.006$ & $104.6 \pm 1.8$ & $108.3 \pm 5.8$ \\
\hline & 19 & 0.49 & $0.0478 \pm 0.0030$ & $0.0165 \pm 0.0003$ & $0.109 \pm 0.007$ & $105.2 \pm 1.9$ & $104.6 \pm 6.7$ \\
\hline & 20 & 0.38 & $0.0476 \pm 0.0033$ & $0.0167 \pm 0.0003$ & $0.109 \pm 0.008$ & $106.5 \pm 2.1$ & $105.3 \pm 7.7$ \\
\hline & 21 & 0.50 & $0.0480 \pm 0.0032$ & $0.0171 \pm 0.0003$ & $0.113 \pm 0.008$ & $109.6 \pm 1.9$ & $109.0 \pm 7.6$ \\
\hline & 22 & 0.50 & $0.0498 \pm 0.0032$ & $0.0169 \pm 0.0003$ & $0.116 \pm 0.008$ & $108.0 \pm 1.8$ & $111.4 \pm 7.5$ \\
\hline & 23 & 0.36 & $0.0475 \pm 0.0032$ & $0.0166 \pm 0.0004$ & $0.108 \pm 0.008$ & $105.9 \pm 2.3$ & $104.5 \pm 7.4$ \\
\hline & 24 & 0.40 & $0.0488 \pm 0.0043$ & $0.0166 \pm 0.0004$ & $0.112 \pm 0.010$ & $105.9 \pm 2.6$ & $107.4 \pm 9.9$ \\
\hline & & & & & Average & $106.7 \pm 0.6$ & $108.6 \pm 1.6$ \\
\hline \multirow[t]{8}{*}{ TG20 } & 1 & 0.77 & $0.0486 \pm 0.0022$ & $0.0164 \pm 0.0003$ & $0.110 \pm 0.006$ & $105.1 \pm 2.1$ & $106.0 \pm 5.3$ \\
\hline & 2 & 0.28 & $0.0509 \pm 0.0031$ & $0.0161 \pm 0.0003$ & $0.113 \pm 0.007$ & $103.1 \pm 2.2$ & $109.0 \pm 7.1$ \\
\hline & 3 & 0.47 & $0.0513 \pm 0.0032$ & $0.0163 \pm 0.0004$ & $0.115 \pm 0.008$ & $104.2 \pm 2.3$ & $110.7 \pm 7.4$ \\
\hline & 4 & 0.26 & $0.0507 \pm 0.0029$ & $0.0157 \pm 0.0004$ & $0.110 \pm 0.007$ & $100.4 \pm 2.7$ & $105.7 \pm 6.7$ \\
\hline & 5 & 0.34 & $0.0492 \pm 0.0020$ & $0.0163 \pm 0.0004$ & $0.110 \pm 0.005$ & $104.1 \pm 2.3$ & $106.3 \pm 4.8$ \\
\hline & 6 & 0.51 & $0.0496 \pm 0.0027$ & $0.0156 \pm 0.0004$ & $0.107 \pm 0.006$ & $100.0 \pm 2.4$ & $103.1 \pm 6.0$ \\
\hline & 7 & 0.53 & $0.0461 \pm 0.0025$ & $0.0162 \pm 0.0004$ & $0.103 \pm 0.006$ & $103.4 \pm 2.4$ & $99.3 \pm 5.9$ \\
\hline & & & & & Average & $103.1 \pm 1.8$ & $105.4 \pm 3.2$ \\
\hline \multirow[t]{17}{*}{ TG29b } & 1 & 0.31 & $0.0474 \pm 0.0037$ & $0.0094 \pm 0.0003$ & $0.061 \pm 0.005$ & $60.3 \pm 1.9$ & $60.5 \pm 5.0$ \\
\hline & 2 & 0.35 & $0.0485 \pm 0.0062$ & $0.0097 \pm 0.0004$ & $0.065 \pm 0.009$ & $61.9 \pm 2.3$ & $63.5 \pm 8.5$ \\
\hline & 3 & 0.32 & $0.0442 \pm 0.0042$ & $0.0098 \pm 0.0003$ & $0.060 \pm 0.006$ & $63.0 \pm 2.1$ & $59.0 \pm 5.9$ \\
\hline & 4 & 0.33 & $0.0469 \pm 0.0049$ & $0.0095 \pm 0.0002$ & $0.062 \pm 0.007$ & $61.1 \pm 1.6$ & $60.7 \pm 6.6$ \\
\hline & 5 & 0.28 & $0.0497 \pm 0.0039$ & $0.0099 \pm 0.0002$ & $0.068 \pm 0.006$ & $63.3 \pm 1.4$ & $66.5 \pm 5.5$ \\
\hline & 6 & 0.77 & $0.0503 \pm 0.0045$ & $0.0098 \pm 0.0002$ & $0.068 \pm 0.006$ & $62.8 \pm 1.5$ & $66.7 \pm 6.2$ \\
\hline & 7 & 0.22 & $0.0497 \pm 0.0042$ & $0.0097 \pm 0.0003$ & $0.067 \pm 0.006$ & $62.5 \pm 1.9$ & $65.6 \pm 5.9$ \\
\hline & 8 & 0.51 & $0.0445 \pm 0.0052$ & $0.0097 \pm 0.0003$ & $0.060 \pm 0.007$ & $62.2 \pm 1.8$ & $58.7 \pm 7.0$ \\
\hline & 9 & 0.27 & $0.0501 \pm 0.0047$ & $0.0097 \pm 0.0003$ & $0.067 \pm 0.007$ & $62.1 \pm 1.8$ & $65.7 \pm 6.4$ \\
\hline & 10 & 0.25 & $0.0472 \pm 0.0041$ & $0.0093 \pm 0.0003$ & $0.061 \pm 0.006$ & $59.7 \pm 1.7$ & $59.6 \pm 5.4$ \\
\hline & 11 & 0.36 & $0.0507 \pm 0.0051$ & $0.0098 \pm 0.0003$ & $0.069 \pm 0.007$ & $63.1 \pm 1.9$ & $67.5 \pm 7.1$ \\
\hline & 12 & 0.34 & $0.0445 \pm 0.0055$ & $0.0097 \pm 0.0003$ & $0.060 \pm 0.008$ & $62.2 \pm 2.2$ & $58.7 \pm 7.5$ \\
\hline & 13 & 0.28 & $0.0473 \pm 0.0046$ & $0.0097 \pm 0.0002$ & $0.063 \pm 0.006$ & $62.0 \pm 1.3$ & $62.1 \pm 6.2$ \\
\hline & 14 & 0.29 & $0.0473 \pm 0.0041$ & $0.0098 \pm 0.0002$ & $0.064 \pm 0.006$ & $63.0 \pm 1.2$ & $62.9 \pm 5.6$ \\
\hline & 15 & 0.26 & $0.0488 \pm 0.0044$ & $0.0094 \pm 0.0002$ & $0.063 \pm 0.006$ & $60.5 \pm 1.3$ & $62.5 \pm 5.8$ \\
\hline & 16 & 0.29 & $0.0462 \pm 0.0038$ & $0.0100 \pm 0.0002$ & $0.063 \pm 0.005$ & $63.9 \pm 1.2$ & $62.5 \pm 5.2$ \\
\hline & & & & & Average & $62.2 \pm 0.7$ & $62.6 \pm 1.5$ \\
\hline
\end{tabular}



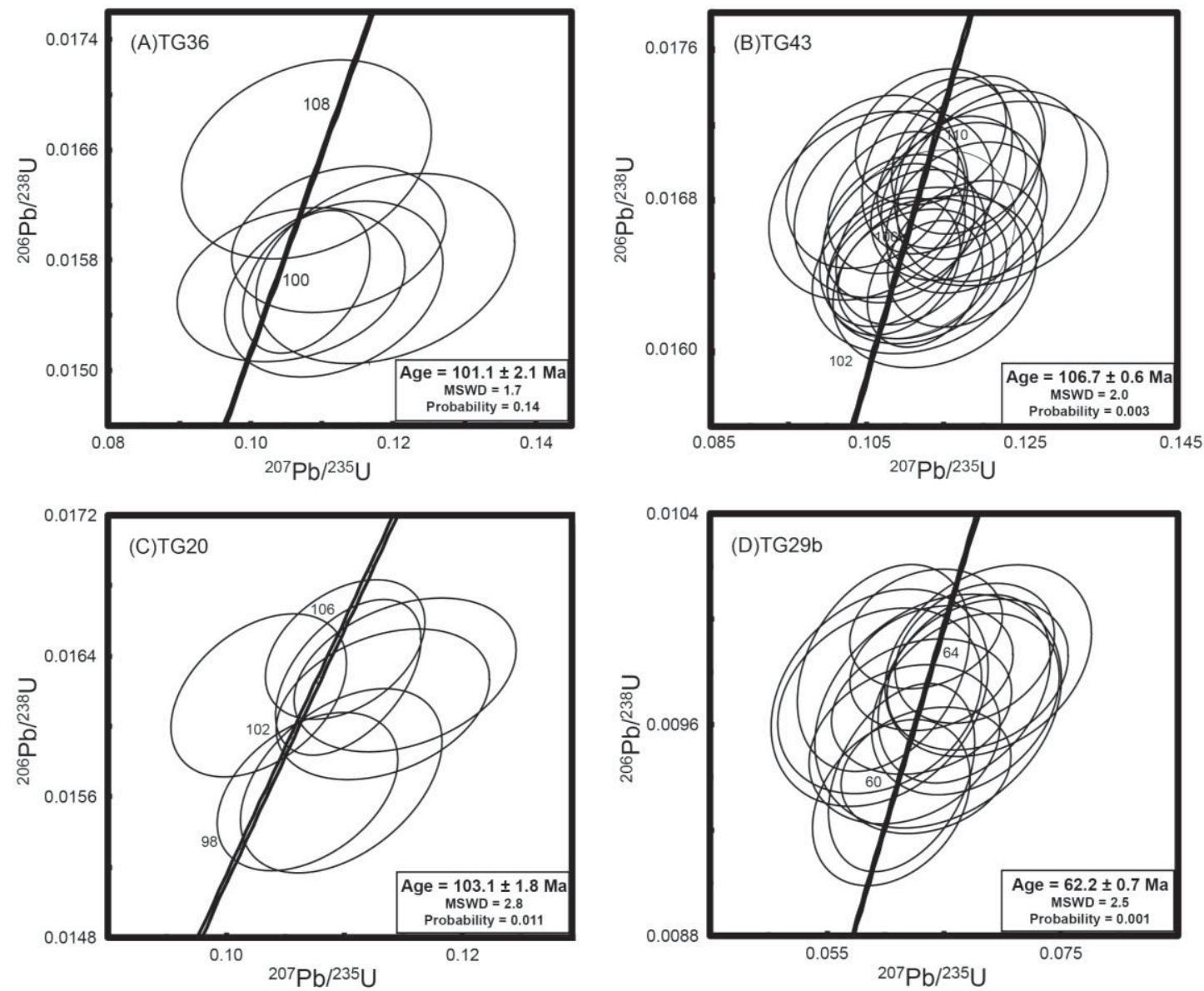

Fig. 8. Concordia diagrams of LA-ICP-MS data for zircons from the Tadamigawa older-stage granites: (A) TG36 of the Hinoematagawa granite from the Taishaku Mountains area, (B) TG43 of the Tadamigawa granite from the Taishaku Mountains area, (C) TG20 of the Tadamigawa granite from the Tadamigawa area, and (D) TG29b of the Tadamigawa granite from the Inagawa area.

than the other Hinoematagawa granite samples. Therefore, it is possible that assimilation of the surrounding sedimentary rocks, especially limestones with relatively high $\mathrm{Sr}$ concentration and high ${ }^{87} \mathrm{Sr} /{ }^{86} \mathrm{Sr}$ ratios, during the very early stage of the granite magma intrusion might disturb the $\mathrm{Sr}$ isotopic homogeneity in a limited part of the Hinoematagawa granite pluton. This is consistent with the field observation that the Jurassic sedimentary Ashio complex in the Taishaku Mountains area consists of a lenticular body of chert, mafic volcanic rocks and limestone, as reported by Yamamoto et al. (2000). Another possibility is that the two samples (TG2,3) might have been produced from different magma than that of the other Hinoematagawa granites.

One sample of the Tadamigawa granite in the Inagawa area (TG29b) deviated largely from the Tadamigawa granite $\mathrm{Rb}$-Sr whole-rock isochron, showing relatively low ${ }^{87} \mathrm{Sr} /{ }^{86} \mathrm{Sr}$ ratios (Table 3). The large deviation indicates that this sample had a different age and/or initial Sr iso- tope ratio than the other Tadamigawa granites and is not genetically related to them.

As for the mineral $\mathrm{Rb}-\mathrm{Sr}$ isochron, although the two samples of TG1a and TG12 yielded concordant ages between the biotite-included and the biotite-excluded isochrons (Table 4, Fig. 6), the three samples of TG5, TG9e and TG13 did not yield concordant ages (Table 4, Fig. 7). The biotite ${ }^{87} \mathrm{Sr} /{ }^{86} \mathrm{Sr}_{\mathrm{i}}$ ratio of the three samples deviated from the biotite-excluded $\mathrm{Rb}$-Sr mineral isochron (Fig. 7), suggesting that the effect of the alteration was limited to biotite. The TG5 and TG13 samples had higher $\mathrm{SiO}_{2}$ contents $(71.4 \mathrm{wt} \%, 72.9 \mathrm{wt} \%)$ than the other samples and the TG9e sample is aplite $\left(\mathrm{SiO}_{2}=76.1 \mathrm{wt} \%\right)$. The three granite samples with high $\mathrm{SiO}_{2}$ contents are considered to have been formed in the last stage of crystal differentiation, which typically accompanies hydrothermal activity. It is possible that the $\mathrm{Rb}-\mathrm{Sr}$ system of the biotite of the three samples (TG5, 9e, 13) had been rejuvenated by hydrothermal alteration. Only the biotite 
Table 6. Geochronological ages of the Tadamigawa older-stage granites

\begin{tabular}{|c|c|c|c|c|c|c|}
\hline \multirow[t]{2}{*}{ Area } & \multirow[t]{2}{*}{ Rock type } & \multirow[t]{2}{*}{ Sample } & \multicolumn{4}{|c|}{ Geochronological data (Ma) } \\
\hline & & & $\mathrm{K}-\mathrm{Ar}(\mathrm{Bt})$ & $\mathrm{U}-\mathrm{Pb}(\mathrm{Zrn})$ & $\mathrm{Rb}-\mathrm{Sr}(\mathrm{WR})$ & $\mathrm{Rb}-\mathrm{Sr}(\mathrm{M})$ \\
\hline \multirow[t]{9}{*}{ Taishaku Mountains area } & Hinoematagawa granite & TG1a & $102.3 \pm 2.2 *$ & & & $97.3 \pm 2.0$ \\
\hline & & TG36 & & $101.1 \pm 2.1$ & & \\
\hline & Tadamigawa granite & TG5 & $102.7 \pm 2.2^{*}$ & & & $95.0 \pm 2.2$ \\
\hline & & TG43 & & $106.7 \pm 0.6$ & & \\
\hline & & & & & $96.5 \pm 1.5$ & \\
\hline & & TG12 & & & & $95.2 \pm 3.8$ \\
\hline & & TG40 & & & & $97.1 \pm 3.1$ \\
\hline & & TG9e & & & & $98.1 \pm 2.9$ \\
\hline & & TG13 & & & & $92.7 \pm 2.7$ \\
\hline Tadamigawa area & Tadamigawa granite & TG20 & $89.6 \pm 1.9 *$ & $103.1 \pm 1.8$ & & \\
\hline Inagawa area & Tadamigawa granite & TG29 & $\begin{array}{l}61.7 \pm 1.4^{*} \\
69 * *\end{array}$ & $62.2 \pm 0.7$ & & \\
\hline
\end{tabular}

*Data from Tanioka et al. (2014). **Data from Kawano and Ueda (1966). Abbreviations are: $K$-Ar (Bt), K-Ar age of biotite; U-Pb (Zrn), ${ }^{238} U$ -

${ }^{206} \mathrm{~Pb}$ age of zircon; $\mathrm{Rb}-\mathrm{Sr}(\mathrm{WR}), \mathrm{Rb}-\mathrm{Sr}$ whole-rock isochron age; $\mathrm{Rb}-\mathrm{Sr}(\mathrm{M}), \mathrm{Rb}$-Sr mineral isochron age. Errors are $2 \sigma$.

plots deviated from the mineral isochrons; however, the closure temperature of biotite in the $\mathrm{Rb}-\mathrm{Sr}$ system $\left(310^{\circ} \mathrm{C}\right.$ : Nishimura and Mogi, 1986) was similar to that of K-feldspar $\left(360^{\circ} \mathrm{C}\right.$ : Nishimura and Mogi, 1986). Some disequilibrium in the $\mathrm{Rb}$-Sr isotope system, such as chloritization, might have occurred only in biotite. Given the reasons above, the biotite-excluded $\mathrm{Rb}$-Sr mineral isochrons were more suitable for estimating the cooling age down to ca. $300^{\circ} \mathrm{C}$ for these three samples (TG5, 9e, 13).

Consequently, the Rb-Sr mineral isochron ages for the Tadamigawa older-stage granites in this study were 97.3 $\pm 2.0 \mathrm{Ma}$ for TG1 a $(\mathrm{SrI}=0.70530 \pm 0.00001, \mathrm{MSWD}=$ 1.3, mineral species: plagioclase, K-feldspar, biotite), 93.5 $\pm 2.8 \mathrm{Ma}$ for TG12 $(\mathrm{SrI}=0.70537 \pm 0.00016, \mathrm{MSWD}=$ 1.2, mineral species: $\mathrm{K}$-feldspar, biotite), $97.1 \pm 3.1 \mathrm{Ma}$ for TG40 ( $\mathrm{SrI}=0.70538 \pm 0.00015$, MSWD $=3.0$, mineral species: plagioclase, $\mathrm{K}$-feldspar), $93.9 \pm 2.7 \mathrm{Ma}$ for TG5 $($ SrI $=0.70533 \pm 0.00001$, MSWD $=0.1$, mineral species: plagioclase, K-feldspar), $98.1 \pm 2.9$ Ma for TG9e $(\mathrm{SrI}=0.70534 \pm 0.00003$, MSWD $=0.5$, mineral species: plagioclase, K-feldspar), and $92.7 \pm 2.7$ Ma for TG13 $(\mathrm{SrI}=0.70545 \pm 0.00004, \mathrm{MSWD}=0.6$, mineral species: plagioclase, K-feldspar) (Figs. 6, 7). The Rb-Sr mineral isochron ages of the Hinoematagawa and Tadamigawa granites in the Taishaku Mountains area coincided approximately with the $\mathrm{Rb}-\mathrm{Sr}$ whole-rock isochron age (96.5 $\pm 1.5 \mathrm{Ma}$ ). These Rb-Sr ages in the Taishaku Mountains area are consistent with the zircon U-Pb ages, and the SrI values from the $\mathrm{Rb}-\mathrm{Sr}$ mineral isochron show the range of $0.7053-0.7055$.

Radiometric dating of the Tadamigawa older-stage granites

The U-Pb and $\mathrm{Rb}-\mathrm{Sr}$ ages analyzed in this study together with the previous reports on biotite $\mathrm{K}-\mathrm{Ar}$ age
(Kawano and Ueda, 1966; Tanioka et al., 2014) for the Tadamigawa older-stage granites are compiled in Table 6. In the Taishaku Mountains area, the zircon U-Pb, Rb$\mathrm{Sr}$ whole-rock isochron and $\mathrm{Rb}-\mathrm{Sr}$ mineral isochron ages all were ca. $100 \mathrm{Ma}$, which were also consistent with the $\mathrm{K}$-Ar ages irrespective of the rock types. These results suggest that the granite in the Taishaku Mountains area was simultaneously formed from a single parental magma and has not suffered a secondary thermal event over the closure temperature of biotite in the $\mathrm{K}$-Ar system $\left(300^{\circ} \mathrm{C}\right.$ : Verschure et al., 1980) after cooling.

The U-Pb age $(106.7 \pm 0.6 \mathrm{Ma})$ of the Tadamigawa granite is slightly older than that $(101.1 \pm 2.1 \mathrm{Ma})$ of the Hinoematagawa granite (Table 6). This age difference is inconsistent with the field relationship that the Hinoematagawa granite has suffered contact metamorphism from the Tadamigawa granite. The age discrepancy in the Taishaku Mountains area pluton might be explained by the differences between the zircon crystallization time and the granitoid emplacement time. More zircon $\mathrm{U}-\mathrm{Pb}$ datings for the Taishaku Mountains area pluton are needed to confirm the age difference.

The central values of the mineral $\mathrm{Rb}$-Sr isochron age show slight variations of the younger age inside (92.793.9 Ma: TG5, 12, 13) and older age outside (97.1-98.1 Ma: TG1a, 9e, 40) within the Taishaku Mountains area pluton (Fig. 2, Table 6); however, the Rb-Sr mineral isochrons have large age errors and are almost the same ages. This could possibly indicate the cooling history of the pluton.

The zircon $\mathrm{U}-\mathrm{Pb}$ age $(106.7 \pm 0.6 \mathrm{Ma})$ of the Tadamigawa granite in the Taishaku Mountains area is older than the biotite $\mathrm{K}$-Ar age $(102.7 \pm 2.2 \mathrm{Ma})$, the $\mathrm{Rb}$ $\mathrm{Sr}$ whole-rock isochron age $(96.5 \pm 1.5 \mathrm{Ma})$ and the $\mathrm{Rb}$ - 
Sr mineral isochron ages (92.7-98.1 Ma) (Table 4). The variation among the zircon $\mathrm{U}-\mathrm{Pb}$ age, the biotite $\mathrm{K}-\mathrm{Ar}$ age and the $\mathrm{Rb}-\mathrm{Sr}$ mineral isochron ages was probably caused by differences in the closure temperatures in these isotope systems $\left(900^{\circ} \mathrm{C}\right.$ for zircon in U-Pb system: Cherniak and Watson, $2001 ; 300^{\circ} \mathrm{C}$ for biotite in the $\mathrm{K}$ Ar system: Verschure et al., $1980 ; 310^{\circ} \mathrm{C}$ for biotite and $360^{\circ} \mathrm{C}$ for K-feldspar in the $\mathrm{Rb}-\mathrm{Sr}$ system: Nishimura and Mogi, 1986). Therefore, the original magma of the Tadamigawa older-stage granites in the Taishaku Mountains area is likely to have gradually cooled within 10 million years.

The $\mathrm{U}-\mathrm{Pb}$ age $(103.1 \pm 1.8 \mathrm{Ma})$ of the Tadamigawa granite (TG20) in the Tadamigawa area is 10 million years older than the K-Ar age $(89.6 \pm 1.9 \mathrm{Ma})$ of the same sample, and the age discrepancy is greater than the limits of analytical uncertainties. The U-Pb age of $c a .100 \mathrm{Ma}$ for the Tadamigawa area agrees with the intrusion age of the Taishaku Mountains area. This indicates that the intrusion age of the Tadamigawa area pluton is $c a .100 \mathrm{Ma}$, which is concurrent with the Taishaku Mountains area pluton. It is likely that a secondary thermal event caused the rejuvenation of the K-Ar system. The closure temperature of zircon in the $\mathrm{U}-\mathrm{Pb}$ system $\left(900^{\circ} \mathrm{C}\right.$ : Cherniak and Watson, 2001) is significantly higher than that of biotite in the $\mathrm{K}-\mathrm{Ar}$ system $\left(300^{\circ} \mathrm{C}\right.$ : Verschure et al., 1980). Therefore, it is possible that the Tadamigawa area pluton was heated over $300^{\circ} \mathrm{C}$ by the intrusion of late Cretaceous basic-felsic dykes after the original formation (Tanioka et al., 2014).

The radiometric ages for the Tadamigawa granite (TG29a, b) in the Inagawa area (ca. $60 \mathrm{Ma}$ ) were significantly younger than the ages of the other areas regardless of the age determination methods (zircon $\mathrm{U}-\mathrm{Pb}$ or biotite $\mathrm{K}$-Ar). The K-Ar age of TG29a $(61.7 \pm 1.4 \mathrm{Ma})$ agreed perfectly with the $\mathrm{U}-\mathrm{Pb}$ age $(62.2 \pm 0.7 \mathrm{Ma})$, which have the high closure temperature of $900^{\circ} \mathrm{C}$. These observations rule out the possibility of the age resetting, and thus the young age corresponds to the intrusion age of the Inagawa area pluton. The slightly older K-Ar age of 69 Ma (Kawano and Ueda, 1966) might be explained by excess Ar. The difference in the formation ages between the Inagawa area pluton and the other area plutons is consistent with the $\mathrm{Rb}-\mathrm{Sr}$ results (SrI). Although both granite plutons in the Taishaku Mountains and the Inagawa area are named en bloc as Tadamigawa granite, these observations indicate that these two plutons have different origins with different intrusion ages and different magma sources.

\section{Origin of the Tadamigawa older-stage granites}

$\mathrm{The} \mathrm{Sr}$ and $\mathrm{Nd}$ isotopic variation of the igneous rocks in the Honshu Arc has been extensively studied (e.g., Kagami et al., 1992, 1999, 2000; Kagami, 2005; Hirahara et al., 2015). The $\mathrm{Sr}$ and $\mathrm{Nd}$ isotopic study of the granitic rocks in the Southwestern Japan Arc revealed that the source composition of the magma changed from a depleted composition (low SrI and high NdI) in the north to an enriched composition (high SrI and low NdI) in the south (Kagami et al., 1992). Additionally, Kagami et al. (1992, 1999) indicated that the origin of these granitic rocks differed between each pluton and can be divided into 5 zones, including the North, South, Transition, Kitakami and Sado Zones, based on the $\mathrm{Sr}$ and Nd isotope compositions (Fig. 1). The initial $\mathrm{Sr}$ and $\mathrm{Nd}$ isotope ratios for the granites in the Northeastern Japan Arc (including the Ashio belt) have been reported to vary from a depleted composition (low SrI and high NdI: the North Zone) in the northeast to an enriched composition (high SrI and low NdI: the South Zone) in the southwest, which is similar to southwestern Japan (Kagami et al., 1999, 2000; Kagami, 2005; Hirahara et al., 2015). The Sr and $\mathrm{Nd}$ isotope compositions of the Transitional Zone of Kagami et al. (1992) corresponds to those between the North and the South Zones or both of the North and South Zones. Additionally, the Kitakami and Sado Zone of Kagami et al. (1999) has been newly classified as an irregular area revealing the different characteristics of 3 zones (the North, South and Transitional Zones). In addition, Hirahara et al. (2015) suggested that the $\mathrm{Sr}$ and $\mathrm{Nd}$ isotopic compositions of granitic rocks in the Northeastern Japan Arc (Abukuma and Asahi granites) and the Ashio belt may correspond to the isotope sections of the Southwestern Japan Arc (the North, South and Transitional Zones). These granites in the Ashio belt and the surrounding rocks have been studied in comparison with the Ryoke granites in southwestern Japan (Yanai, 1972, 1973a, 1973b). The study of the Quaternary volcanic rocks in northeastern Japan also revealed that the magma characteristics of volcanic rocks have relationships with the surrounding crustal materials, such as granite (Kimura and Yoshida, 2006; Tatsumi et al., 2008; Takahashi et al., 2013). There are four causes of the diversity of the initial isotope ratios for these granites: 1) the difference in mixing rates with the crustal materials (Shibata and Ishihara, 1979), 2) the regional heterogeneity of the upper mantle as source materials (Terakado and Nakamura, 1984), 3) the difference in contamination by slab components derived from the subducted sedimentary rocks (Takagi, 2004), and 4) the contribution by the upper mantle or the lower crustal materials (Kagami et al., 1999).

The Tadamigawa older-stage granites were compared with the granites in the Yamizo Mountains area. The granites in the Yamizo Mountains area can be divided into two types: the older pluton (ca. $105 \mathrm{Ma}$ : Shibata et al., 1973 ) and the younger pluton (ca. 65 Ma: Kawano and Ueda, 1966). The formation ages of these rocks are concordant with those of the Tadamigawa older-stage 


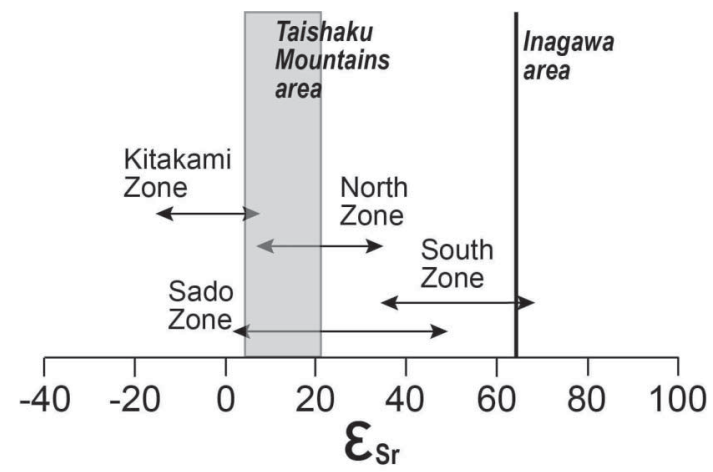

Fig. 9. $\varepsilon_{S r}$ diagram. Fields of the Kitakami, North, South and Sado Zones are from Kagami et al. (1992), Kagami et al. (1999), Kagami et al. (2000), and Kagami (2005). Shaded area indicates the initial ${ }^{87} \mathrm{Sr} /{ }^{86} \mathrm{Sr}$ ratio of the Tadamigawa older-stage granites in the Taishaku Mountains area, and a single line indicates the initial ${ }^{87} \mathrm{Sr} /{ }^{86} \mathrm{Sr}$ ratio of the Tadamigawa granite in the Inagawa area.

granites. The SrI of the Tadamigawa granite in the Inagawa area was estimated as 0.70894 using the presentday whole-rock ${ }^{87} \mathrm{Sr} /{ }^{86} \mathrm{Sr}$ ratio of 0.722118 (TG29b) and the zircon U-Pb ages of $60 \mathrm{Ma}$. Kagami et al. (2000) reported that the initial $\mathrm{Sr}$ and $\mathrm{Nd}$ isotope ratios of the granites in the Yamizo Mountains differed significantly between the older pluton and the younger pluton, and these rocks belong to the Transitional Zone from these isotope ratios. The older pluton corresponds to the North Zone as well as the Abukuma Mountains, and the younger pluton corresponds to the South Zone as well as the Ashio Mountains. The $\varepsilon_{\mathrm{Sr}}$ value at $100 \mathrm{Ma}$ for each whole-rock sample in the Taishaku Mountains and that at $60 \mathrm{Ma}$ in the Inagawa area were calculated with the parameters for Bulk Earth, $\left.\left({ }^{87} \mathrm{Sr} /{ }^{86} \mathrm{Sr}\right)\right)_{\mathrm{pr}}=0.7045$ and ${ }^{87} \mathrm{Rb} /{ }^{86} \mathrm{Sr}=0.0827$ (DePaolo, 1988). The $\varepsilon_{\mathrm{Sr}}$ values of the Tadamigawa granite in the Taishaku Mountains area range from +4.6 to +20.4 and are concordant with the range of the North Zone of Kagami et al. (1992) (Fig. 9). The $\varepsilon_{\mathrm{Sr}}$ value of the Inagawa area $(60 \mathrm{Ma})$ is +64.0 , and is concordant with the range of the South Zone of Kagami et al. (1992). The $\varepsilon_{\mathrm{Sr}}$ values of the Tadamigawa older-stage granites in the Taishaku Mountains and Inagawa areas were compared with the older and younger plutons in the Yamizo Mountains, respectively. The results show the clear differences in the source magmas between the Taishaku Mountains and Inagawa areas. The Tadamigawa older-stage granites also show the characteristics of the Transitional Zone with the isotope compositions of both the North and South Zones.

The relatively constant $\varepsilon_{\mathrm{Sr}}$ values $(+4.6-+20.4)$ of the Tadamigawa older-stage granites in the Taishaku Mountains together with the major and trace element com- positions (Figs. 3 and 4) indicate the fractional differentiation from the same source materials. Meanwhile, a local scale heterogeneity of the $\varepsilon_{\mathrm{Sr}}$ value was also found in the plutons. For example, the $\varepsilon_{\mathrm{Sr}}$ values $(+20.0,+20.4)$ of the Hinoematagawa granites (TG2, 3) are higher than those $(+4.6-+13.8)$ of the other granites in the Taishaku Mountains area. The higher $\varepsilon_{\mathrm{Sr}}$ values for the Tadamigawa older-stage granites in the Taishaku Mountains area (TG2, 3) probably reflect assimilation of the surrounding sedimentary rocks, that is the mixing of crustal materials. This is supported by the field observation as already stated in the section on the $\mathrm{Rb}-\mathrm{Sr}$ whole-rock isochron ages.

\section{Conclusion}

The U-Pb ages of zircon for the Tadamigawa olderstage granites in the Taishaku Mountains area were 101.1 $\pm 2.1 \mathrm{Ma}$ for the Hinoematagawa granite (TG36) and 106.7 \pm 0.6 Ma for the Tadamigawa granite (TG43), and the $\mathrm{Rb}-\mathrm{Sr}$ whole-rock isochron age for the Tadamigawa granite was $96.5 \pm 1.5 \mathrm{Ma}$ in the Taishaku Mountains area. The $\mathrm{U}-\mathrm{Pb}$ ages of zircon were $103.1 \pm 1.8 \mathrm{Ma}$ for the Tadamigawa granite (TG20) in the Tadamigawa area and $62.2 \pm 0.7 \mathrm{Ma}$ for the Tadamigawa granite (TG29b) in the Inagawa area. The intrusion ages for the Tadamigawa older-stage granites were $c a$. $100 \mathrm{Ma}$ for the Taishaku Mountains and the Tadamigawa areas and $c a$. $60 \mathrm{Ma}$ for the Inagawa area according to the radiometric ages for each pluton (Kawano and Ueda, 1966; Tanioka et al., 2014; and this study). The K-Ar age of $89.6 \pm 1.9$ Ma in the Tadamigawa area was interpreted as the rejuvenation age, which is caused by the reheating of the intrusion of the surrounding dykes. In addition, the radiometric ages (ca. $60 \mathrm{Ma}$ ) in the Inagawa area pluton corresponded not to the rejuvenated age but rather to the formation age of the Inagawa area pluton.

Additionally, the $\mathrm{Rb}-\mathrm{Sr}$ mineral isochron ages of the Hinoematagawa and the Tadamigawa granites in the Taishaku Mountains area show ages of 92.7-98.1 Ma, and the ages are concordant with the $\mathrm{Rb}-\mathrm{Sr}$ whole-rock isochron age $(96.5 \pm 1.5 \mathrm{Ma})$. This indicates that the Hinoematagawa and the Tadamigawa granites in the Taishaku Mountains area were formed by the differentiation of the single parental magma.

The $\varepsilon_{\mathrm{Sr}}$ values of the Tadamigawa older-stage granites are $+4.6-+20.4$ in the Taishaku Mountains area (100 $\mathrm{Ma})$ and +64.0 in the Inagawa area $(60 \mathrm{Ma})$. The Tadamigawa older-stage granites comprise plutons with different ages and origins. The $\varepsilon_{\mathrm{Sr}}$ values of the Tadamigawa older-stage granites in the Taishaku Mountains area are concordant with that of the North Zone of Kagami et al. (1992), and the value in the Inagawa area is concordant with that of the South Zone. The two plutons for the Tadamigawa older-stage granites have different 
origins with different formation ages and different magma sources; however, the cause of the change in magma source according to age is still unclear. Measurement of multiple isotopes such as $\mathrm{Nd}, \mathrm{Pb}$ and $\mathrm{Hf}$ for the Tadamigawa older-stage granites in northeastern Japan is needed to determine the cause of the differences in magma sources of the igneous rocks across the ages. Additionally, field and microscope observation are indispensable for additional explanation.

Acknowledgments - We thank Prof. K. Yamamoto and Ms. M. Shimizu for their help with the U-Pb isotope analyses at Nagoya University and the Marine Works Japan staffs for their help during the laboratory experiments at KCC. We would also like to thank Prof. M. Minami for her assistance at Nagoya University. This work was supported by KAKENHI (24540527, 16K05623).

\section{REFERENCES}

Anders, E. and Grevesse, N. (1989) Abundances of the elements: meteoritic and solar. Geochim. Cosmochim. Acta 53, 197214.

Bea, F. (1996) Residence of REE, Y, Th and U in granites and crustal protoliths; implications for the chemistry of crustal melts. J. Petrol. 37(3), 521-552.

Cherniak, D. J. and Watson, E. B. (2001) Pb diffusion in zircon. Chem. Geol. 172, 5-24.

Chihara, K. and Komatsu, M. (1992) Geology of the Hakkaisan district. With geological Sheet Map at 1:50,000. Geol. Surv. Japan, 107 pp. (in Japanese with English abstract 8 pp.).

DePaolo, D. J. (1988) Age dependence of the composition of continental crust: evidence from $\mathrm{Nd}$ isotopic variations in granitic rocks. Earth Planet. Sci. Lett. 90(3), 263-271.

Hirahara, Y., Senda, R., Takahashi, T., Tsuchiya, N., Kagami, S., Yoshida, T., Chang, Q., Miyazaki, T., Bogdan, S. V. and Kimura, J.-I. (2015) Spatial variation of Sr-Nd-Hf isotopic compositions in from Cretaceous to Paleogene granitoids from Northeastern Japan Arc. Japan. Mag. Mineral. Petrol. Sci. 44, 91-111 (in Japanese with English abstract).

Horn, I. and Blanckenburg, F. V. (2007) Investigation on elemental and isotopic fractionation during $196 \mathrm{~nm}$ femtosecond laser ablation multiple collector inductively coupled plasma mass spectrometry. Spectrochim. Acta B 62 , 410-422.

Kagami, H. (2005) Formative periods and source materials of Cretaceous-Paleogene granitoids from Honshu Arc. Jour. Geol. Soc. Japan 111, 441-457 (in Japanese with English abstract).

Kagami, H., Iizumi, S., Tainosho, Y. and Owada, M. (1992) Spatial variations of $\mathrm{Sr}$ and $\mathrm{Nd}$ isotope ratios of CretaceousPaleogene granitoid rocks, Southwest Japan Arc. Contrib. Mineral. Petrol. 112, 165-177.

Kagami, H., Kawano, Y., Ikawa, T., Ishioka, J., Kagashima, S., Yuhara, M., Shuto, K., Iizumi, S., Imaoka, T., Owada, M., Osanai, Y. and Tinosho, Y. (1999) Transition of space and time of Cretaceous to Tertiary igneous activity and lower crust of Honshu Arc-examination based on Rb-Sr whole rock isochron ages and $\mathrm{Sr}$ and $\mathrm{Nd}$ isotopes. Mem. Geol. Soc. Japan 53, 1-19 (in Japanese with English abstract).

Kagami, H., Kawano, Y., Ikawa, T., Ishioka, J., Kagashima, S., Shimura, T., Shuto, K., Iizumi, S., Imaoka, T., Owada, M., Osanai, Y., Tainosho, Y., Tanaka, H., Tsuchiya, N. and Yuhara, M. (2000) Regional variations of initial Sr and Nd isotope ratios of late Cretaceous to Paleogene granitoid rocks, Honshu arc. Chikyu Monthly, Special Issue 30, 185190 (in Japanese).

Kawano, Y. and Ueda, Y. (1965a) K-Ar dating on the igneous rocks in Japan (II)_Granitic rocks in Kitakami massif-. Jour. Mineral. Petrol. Econ. Geol. 53, 143-154 (in Japanese with English abstract).

Kawano, Y. and Ueda, Y. (1965b) K-Ar dating on the igneous rocks in Japan (III)_Granitic rocks in Abukuma massif-. Jour. Mineral. Petrol. Econ. Geol. 54, 162-172 (in Japanese with English abstract).

Kawano, Y. and Ueda, Y. (1966) K-Ar dating on the igneous rocks in Japan (IV)_Granitic rocks in northeastern Japan-. Jour. Mineral. Petrol. Econ. Geol. 56, 41-55 (in Japanese with English abstract).

Kimura, J.-I. and Yoshida, T. (2006) Contributions of slab fluid, mantle wedge and crust to the origin of quaternary lavas in the NE Japan arc. J. Petrol. 47, 2185-2232.

Kinoshita, O. and Ito, H. (1990) Reconstruction of southwest Japan and northeast Japan based on trends of Mesozoic igneous rock ages. J. Geol. Soc. Japan 96, 821-838 (in Japanese with English abstract).

Kouchi, Y., Obara, H., Fujimoto, T., Orihashi, Y., Haruta, Y. and Yamamoto, K. (2015) Zircon U-Pb dating by $213 \mathrm{~nm}$ Nd: YAG laser ablation inductively coupled plasma mass spectrometry: Optimization of the analytical condition to use NIST SRM 610 for $\mathrm{Pb} / \mathrm{U}$ fractionation correction. Chikyukagaku (Geochemistry) 49, 19-35 (in Japanese with English abstract).

Ludwig, K. R. (2008) User's manual for Isoplot 3.70. Berkeley Geochronological Center Special Publication 4, 77 pp.

Murayama, M. and Kawata, K. (1955) 1:50,000 Geological Map of Japan, Hiuchi-Dake with Explanatory Text. Geol. Surv. Japan, 32 pp. (in Japanese with English abstract).

Nakajima, T., Shirahase, T. and Shibata, K. (1990) Along-arc lateral variation of $\mathrm{Rb}-\mathrm{Sr}$ and $\mathrm{K}-\mathrm{Ar}$ ages of Cretaceous granitic rocks in Southwest Japan. Contrib. Mineral. Petrol. 104(4), 381-389.

Nakazaki, M., Tsuboi, M., Kanagawa, K., Kato, T. and Suzuki, K. (2004) Quantitative chemical analysis of rocks with Xray fluorescence analyzer XRF-1800. Bull. Nagoya Univ. Museum 20, 79-91.

Nishimura, S. and Mogi, T. (1986) The interpretation of discordant ages of some granitic bodies. Jour. Geotherm. Res. Soc. Japan 8(3), 145-164.

Noda, A., Tsuboi, M., Kurihara, T. and Uchino, T. (2014) The Permian and Jurassic strata around the border between Niigata and Fukushima prefectures. Annual Meeting of the Geological Society of Japan The 121st Annual Meeting (2014' Kagoshima), Geol. Soc. Japan, 229 pp. (in Japanese); doi:10.14863/geosocabst.2014.0_444.

Nozawa, T. (1975) Granite and asociated rocks. An Outline of the Geology of Japan (Yoshida, T., ed.), 3rd ed., Geol. Surv. 
Japan, 24-30.

Orihashi, Y., Nakai, S. and Hirata, T. (2008) U-Pb age determination for seven standard zircons using inductively coupled plasma-mass spectrometry coupled with frequency quintupled Nd-YAG $(\lambda=213 \mathrm{~nm})$ laser ablation system: comparison with LA-ICP-MS zircon analyses with a NIST glass reference material. Resour. Geol. 58(2), 101-123.

Otsuki, K. and Ehiro, M. (1978) Major strike-slip faults and their bearing on spreading in the Japan Sea. Jour. Phys. Earth 26 (Suppl.), S537-S555.

Sato, H. and Amano, K. (1991) Relationship between tectonics, volcanism, sedimentation and basin development, Late Cenozoic, central part of Northern Honshu, Japan. Sed. Geol. 74(1-4), 323-343.

Shibata, K. (1966) K-Ar ages of the Nihonkoku gneiss, northeast Japan. Bull. Geol. Surv. Japan 17, 426-429.

Shibata, K. and Ishihara, S. (1979) Initial ${ }^{87} \mathrm{Sr} /{ }^{86} \mathrm{Sr}$ ratios of plutonic rocks from Japan. Contrib. Mineral. Petrol. 70(4), 381-390.

Shibata, K. and Miller, J. A. (1962) Potassium-Argon ages of granitic rocks from the Kitakami highlands. Bull. Geol. Surv. Japan 13(8), 709-711.

Shibata, K. and Utsumi, S. (1983) K-Ar ages on hornblendes from granitic rocks in the southern Abukuma Plateau. Japan. Mineral. Petrol. Econ. Geol. 78, 405-410 (in Japanese with English abstract).

Shibata, K., Hachisu, T. and Uchiumi, S. (1973) K-Ar ages of granitic rocks from the Yamizo Mountains. Bull. Geol. Surv. Japan 24, 513-518 (in Japanese with English abstract).

Shinotsuka, K., Hidaka, H. and Ebihara, M. (1995) Detailed abundances of rare earth elements, thorium and uranium in chondritic meteorites: An ICP-MS study. Meteoritics 30(6), 694-699.

Steiger, R. H. and Jäger, E. (1977) Subcommission on geochronology: convention on the use of decay constants in geo- and cosmochronology. Earth Planet. Sci. Lett. 36, $359-362$.

Sudo, M., Uto, K., Anno, K., Ishizuka, O. and Uchiumi, S. (1998) SORI93 biotite: A new mineral standard for K-Ar dating. Geochem. J. 32, 49-58.

Takagi, T. (2004) Origin of magnetite-and ilmenite-series granitic rocks in the Japan arc. Amer. Jour. Sci. 304(2), 169202.

Takahashi, T., Hirahara, Y., Miyazaki, T., Senda, R., Chang, Q., Kimura, J.-I. and Tatsumi, Y. (2013) Primary magmas at the volcanic front of the NE Japan arc: coeval eruption of crustal low-K tholeiitic and mantle-derived medium-K calc-alkaline basalts at Azuma volcano. J. Petrol. 54, 103148.

Takahashi, Y., Toyoshima, T., Shimura, T., Hara, H., Takeuchi, K., Sakai, A. and Nakano, S. (2005) Geological map of Japan 1:50,000, Suhara. Chishitsu News 607, 57-62 (in Japanese).
Tanioka, Y., Wakasugi, Y. and Tsuboi, M. (2014) Whole-rock chemical compositions and K-Ar ages of the Tadamigawa granitic rocks, southwestern part of Fukushima Prefecture, northeastern Japan. Japan. Mag. Mineral. Petrol. Sci. 43, 215-227 (in Japanese with English abstract).

Tatsumi, Y., Takahashi, T., Hirahara, Y., Chang, Q., Miyazaki, T., Kimura, J.-I., Ban, M. and Sakayori, A. (2008) New insights into andesite genesis: the role of mantle-derived calc-alkalic and crust-derived tholeiitic melts in magma differentiation beneath Zao volcano, NE Japan. J. Petrol. 49, 1971-2008.

Terakado, Y. and Nakamura, N. (1984) Nd and Sr isotopic variations in acidic rocks from Japan: significance of uppermantle heterogeneity. Contrib. Mineral. Petrol. 87, 407417.

Ueno, N. (1977) Rb-Sr and K-Ar isotopic variations of granodiorites and associated metamorphic rocks in the Abukuma Plateau, Japan. Earth Sci. (Chikyu Kagaku) 31, 49-54.

Verschure, R. H., Andriessen, P. A. M., Boelrijk, N. A. I. M., Hebeda, E. H., Maijer, C., Priem, H. N. A. and Verdurmen, E. T. (1980) On the thermal stability of Rb-Sr and K-Ar biotite system: evidence from coexisting Sveconorwegian (ca $870 \mathrm{Ma}$ ) and Caledonian (ca $400 \mathrm{Ma}$ ) biotites in SW Norway. Contrib. Mineral. Petrol. 74, 245-252.

Wakaki, S. and Tanaka, T. (2005) Single mineral Rb-Sr isochron dating applied to the Nohi Rhyolite and a quartz porphyry dyke, central Japan. Geochem. J. 39, 21-28.

Wakaki, S., Obata, H., Tazoe, H. and Ishikawa, T. (2017). Precise and accurate analysis of deep and surface seawater $\mathrm{Sr}$ stable isotopic composition by double-spike thermal ionization mass spectrometry. Geochem. J. 51(3), 227-239.

Wiedenbeck, M., Allé, P., Corfu, F., Griffin, W. L., Meier, M., Oberli, F., Quadt, A. V., Roddick, J. C. and Speigel, W. (1995) Three natural zircon standards for U-Th-Pb, Lu-Hf, trace element and REE analyses. Geostand. Newsl. 19, 123.

Yamamoto, T., Takizawa, F., Takahashi, F., Kubo, K., Komazawa, M., Hiroshima, T. and Sudo, S. (2000) Geological Map of Japan 1:200,000, Nikko. Geol. Surv. Japan (in Japanese with English abstract).

Yanai, K. (1972) The late Mesozoic acidic igneous rocks of the northern Ashio Mountainland Part 1. Field geology. Jour. Mineral. Petrol. Econ. Geol. 67, 193-207 (in Japanese with English abstract).

Yanai, K. (1973a) The late Mesozoic acidic igneous rocks of the northern Ashio Mountains. Part 2. Petrography. Jour. Mineral. Petrol. Econ. Geol. 68, 6-29 (in Japanese with English abstract).

Yanai, K. (1973b) The late Mesozoic acidic igneous rocks of the northern Ashio Mountains Part 3. Petrogenesis. Jour. Mineral. Petrol. Econ. Geol. 68, 78-86 (in Japanese with English abstract). 